\title{
How cells engulf: a review of theoretical approaches to phagocytosis
}

\author{
David M. Richards ${ }^{1,2, *}$, Robert G. Endres ${ }^{2}$ \\ ${ }^{1}$ Centre for Biomedical Modelling and Analysis, Living Systems Institute, \\ University of Exeter, Exeter, EX4 4QD, UK \\ ${ }^{2}$ Department of Life Sciences, Imperial College, London, SW7 2AZ, UK \\ * E-mail: david.richards@exeter.ac.uk
}

\begin{abstract}
Phagocytosis is a fascinating process whereby a cell surrounds and engulfs particles such as bacteria and dead cells. This is crucial both for single-cell organisms (as a way of acquiring nutrients) and as part of the immune system (to destroy foreign invaders). This whole process is hugely complex and involves multiple coordinated events such as membrane remodelling, receptor motion, cytoskeleton reorganisation and intracellular signalling. Because of this, phagocytosis is an excellent system for theoretical study, benefiting from biophysical approaches combined with mathematical modelling. Here, we review these theoretical approaches and discuss the recent mathematical and computational models, including models based on receptors, models focusing on the forces involved, and models employing energetic considerations. Along the way, we highlight a beautiful connection to the physics of phase transitions, consider the role of stochasticity, and examine links between phagocytosis and other types of endocytosis. We cover the recently discovered multistage nature of phagocytosis, showing that the size of the phagocytic cup grows in distinct stages, with an initial slow stage followed by a much quicker second stage starting around half engulfment. We also address the issue of target shape dependence, which is relevant to both pathogen infection and drug delivery, covering both one-dimensional and two-dimensional results. Throughout, we pay particular attention to recent experimental techniques that continue to inform the theoretical studies and provide a means to test model predictions. Finally, we discuss population models, connections to other biological processes, and how physics and modelling will continue to play a key role in future work in this area.
\end{abstract}

\section{Contents}

1 Introduction $\quad 2$

1.1 Review outline .................... . . . 3

1.2 Why phagocytosis? ................ 4 
1.3 The biology of phagocytosis . . . . . . . . . . . 5

1.4 Phagocytosis in the immune system . . . . . . . . . . 8

1.5 Physics, mathematics and modelling . . . . . . . . . . 9

2 Receptor models $\quad 10$

2.1 Phagocytic receptors . . . . . . . . . . . . . . . . . . 10

2.2 Receptor models in endocytosis . . . . . . . . . . . . . . . . 11

2.3 Receptor models in phagocytosis . . . . . . . . . . . . . . 14

2.4 The effect of signalling . . . . . . . . . . . . . . . . 17

3 Force and energy models $r$

3.1 Dual-micropipette experiments . . . . . . . . . . . . . . . . . 19

3.2 Force-based models . . . . . . . . . . . . . . . . . . 20

3.3 Energy-based models . . . . . . . . . . . . . . . . 24

4 Stages of engulfment $\quad 25$

5 Target shape dependence $\quad 27$

5.1 Experimental results . . . . . . . . . . . . . . . . . . 28

5.2 Highly-symmetric targets . . . . . . . . . . . . . . . . . . . . . 29

5.3 Lower symmetry targets . . . . . . . . . . . . . . . . . 32

$6 \quad$ Future directions $\quad 34$

$\begin{array}{lll}7 & \text { Outlook } & 36\end{array}$

\section{Introduction}

Cells often need to bring large particles into the cell interior. For example, this is used to destroy pathogens such as bacteria and to remove dead cells. This process, called phagocytosis from the Ancient Greek for "eating" and "cell", requires transporting particles through the cell membrane, even though the membrane is purposely constructed to act as a barrier to all but the smallest particles. Cells circumvent this problem by wrapping themselves around the target particle and creating a new internal membrane-bound compartment. As such, during phagocytosis, particles never actually cross the membrane.

In part due to the difficulty in engulfing such large particles, phagocytosis is a fabulously complex process that involves multiple, coordinated cellular processes such as remodelling of the cell membrane, rearrangement of the cytoskeleton, and extensive signalling cascades $[1,2]$. This may explain why 
there have been relatively few theoretical models, with the fear that little will be learned without including every component. However, recent work has shown that this need not be the case, and that important progress can still be made by focusing on simple models that include only a few key ingredients [3].

This review will describe these theoretical advances, which include ideas from membrane physics, reaction-diffusion systems, force-based modelling and signalling networks. In each case, close connection with experiment has been essential and it is necessary to understand some of these experimental set-ups to fully appreciate the advances made. This has meant that phagocytosis has joined the increasing number of biological and medical areas where the application of ideas from physics, combined with mathematical and computational modelling, is enabling novel understanding at a pace that could not be matched from purely experimental approaches.

\subsection{Review outline}

Since there is often a perceived hurdle to understanding the fundamental concepts of phagocytosis, we first introduce the basic biological processes involved, including a brief overview of the immune system, the cell membrane, the cytoskeleton and intracellular signalling. This will be followed by an introduction to the role that modelling approaches can play, both in phagocytosis and in related areas.

We then first describe models that focus on the motion of receptors (membrane-bound proteins that recognise foreign elements). These typically involve reaction-diffusion systems with additional components to describe the cytoskeleton and signalling. We will explain how, in some formulations, the whole phagocytic engulfment process can be understood as a phase transition between bound and unbound receptors.

Next, we describe models that focus either on the physical forces involved or on energetic considerations. These approaches typically include concepts such as membrane tension, membrane curvature, flattening forces and volume conservation. These models are intimately connected with dual-micropipette experiments, which we explain in detail. These experiments are providing the necessary high-resolution time-lapse movies that are driving forward the joint modelling-experimental approaches to understanding phagocytosis.

Various advances that have directly arisen by applying modelling to phagocytosis are covered next. These include the multistage nature of engulfment (with an initial slow stage followed by a much quicker second stage) and the dependence on target shape, size and orientation. These advances require extensions to two-dimensional membrane models that are capable of describing 
more-realistic, lower-symmetry arrangements.

Finally, we discuss some of the numerous future directions that this field is likely to take. These include the role of stochasticity, connections with other types of endocytosis, differences between various types of phagocytosis, and the effect of the target stiffness and coating. We also briefly touch on other aspects of phagocytosis where modelling will also be valuable in the future, such as the motion of the phagosome within the cell once engulfment is complete.

\subsection{Why phagocytosis?}

Phagocytosis is used by many different organisms. These include single-cell organisms (such as Dictyostelium, Entamoeba and ciliates), where phagocytosis is used to acquire nutrients, and organisms with complex immune systems, where it is a method of both destroying foreign elements (such as bacteria) and removing cell debris (such as apoptosed cells). That such a broad range of organisms use the same mechanism is a result of the ancient origin of phagocytosis [4-6]. Consequently, determining the similarities and differences between phagocytosis in various organisms can shed light on the evolutionary history of endocytosis and on how innate and adaptive immunity evolved.

Due to the central role it plays in our immune system, understanding phagocytosis has widespread medical applications, both in health and disease. For example, immune cells continuously identify and phagocytose bacteria, which leads to a multitude of questions about how the composition, stiffness, size and shape of pathogens affects the success of phagocytosis. Of course, such bacteria do not sit idly by and wait to be destroyed, but rather continually try to avoid engulfment by, for example, controlling their orientation and surface composition, leading to an ongoing battle with the immune system [7]. For example, pathogenic E. coli secrete proteins to inhibit phagocytic uptake [8-10]. Further, some bacteria, such as Mycobacterium tuberculosis [11] and Salmonella typhi [12], actively want to be phagocytosed in order to gain entry to cells. In such cases, it would actually be advantageous for the immune system to fail in phagocytosis.

Various medical conditions are related to normal or defective forms of phagocytosis. For example, Wiskott-Aldrich syndrome is an X-linked disease that is connected with phagocytic impairment and is characterised by immune deficiency, eczema and low platelet count [13-16]. This condition is caused by a mutation in WASP, a protein involved (via activation of the Arp2/3 complex) in branching of actin chains, which in turn is important in forming and contracting the phagocytic cup $[17,18]$. As another example, tu- 
mours often evade destruction by expressing "don't-eat-me" signals on their surfaces. These signals prevent immune cells recognising and phagocytosing the tumour cells [19]. Finding methods for interfering with these signals, or even expressing "eat-me" signals, could lead to more effective treatments for cancer [20].

Micro- and nanoparticle drug carriers are an increasingly promising avenue for combating a range of diseases, including HIV [21], TB [22] and cancer $[23,24]$. Potential advantages of such drugs include protection from degradation, fewer side effects on vital tissues, and smaller doses [25,26]. Further, by placing drug carriers inside immune cells, it is potentially possible to target drugs directly to where they are needed (such as the site of infection or a tumour) [27]. One problem with this approach is that microparticle drug carriers are often identified as foreign particles by the immune system and removed via phagocytosis $[28,29]$. Solving this problem, perhaps by careful design of the microparticles and better understanding of phagocytosis, could lead to huge impact for human health.

The basic biological and physical processes involved during phagocytosis, such as changing membrane shape, remodelling of the cytoskeleton, movement of proteins to specialised sites, and signalling cascades, are relevant to various related processes. These include other forms of endocytosis [30], chemotaxis [31], and immunological synapse formation [32]. This means that progress and modelling in phagocytosis may well be adaptable to other processes within the immune system. This is especially true since many recent models of phagocytosis focus on only the most fundamental biophysical mechanisms, which are likely to be similar across closely related biological processes.

\subsection{The biology of phagocytosis}

Nature has evolved a vast range of mechanisms for transporting substances across cell membranes. Although this can sometimes happen passively (such as via osmosis and diffusion), active transport (using, for example, electrochemical gradients or specialised membrane pumps) is often required. Relatively large particles or volumes of fluid can be moved using a particular type of active transport that involves the creation of small membrane compartments. This is called either endocytosis when material is brought inside cells, or exocytosis when internal material is moved to the outside.

Endocytosis, which has many similarities with exocytosis, involves first reshaping the cell membrane to form a pocket containing the external material. This pocket then gradually pinches off to leave an internal membranebound compartment called a vesicle. This fascinating process occurs in a 


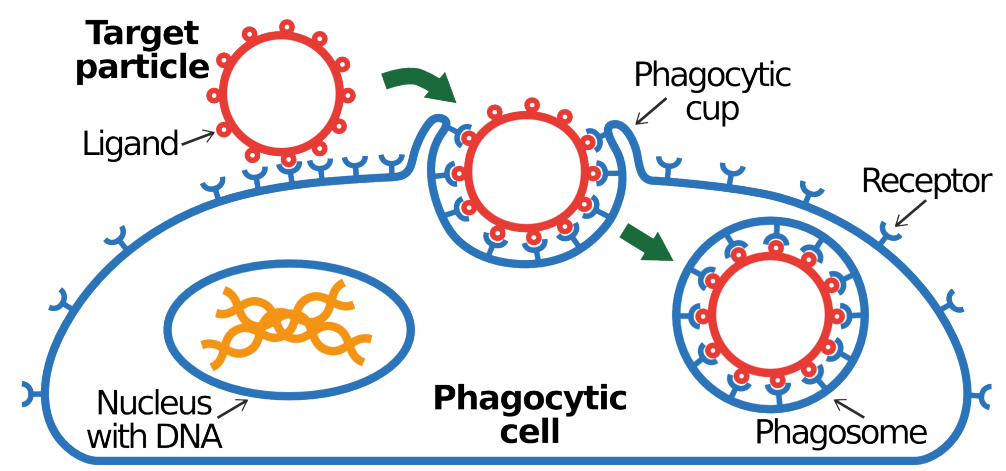

Figure 1. The basic process of phagocytosis. Receptors in the cell membrane attach to ligands on the target particle. Bound receptors signal to the rest of the cell, which recruits actin and causes the membrane to gradually wrap around the target forming the phagocytic cup. Once the membrane closes around the top/end of the particle, the target is completely within the cell in a membrane-bound compartment called the phagosome.

number of different forms, based on vesicle size, how exactly the cell membrane behaves, and the proteins involved. Although there is little consensus on the exact number of distinct forms [30], endocytosis is often split into four types. The first two, clathrin-mediated and caveolar-type endocytosis, involve relatively small vesicles (typically $<100 \mathrm{~nm}$ ) and use coated membrane pits or cave-shaped invaginations. The third type, macropinocytosis, is used for internalising fluid and so is often called "cellular drinking". The final type (the focus of this review) is phagocytosis, which involves internalisation of relatively large solid particles.

Phagocytosis, typically defined as engulfment of particles over $0.5 \mu \mathrm{m}$ but which can involve particles up to $20 \mu \mathrm{m}$ or even larger [33], was first studied by Élie Metchnikoff in the 1880s [34,35], with the first live-cell movies recorded by Jean Comandon around $1910[36,37]$. The process can be split into a number of different stages: target recognition, wrapping of the cell around the target, creation of the phagosome, and processing of the phagosome.

The first stage, target recognition, involves receptors in the cell membrane (Fig. 1). These are specialised proteins that recognise and bind to the target particle, either directly or via opsonins (molecules such as antibodies and complement proteins that bind to the target and enhance phagocytosis). The component of the target that is bound (called the ligand) is now physically linked to the cell. In order to deal with the huge range of different target types, there are a multitude of different receptors each having various 
specificities for a number of different ligands [38-40]. Typically, more than one receptor type will be involved in identifying and phagocytosing a given target.

Bound receptors signal to the rest of the cell and so initiate the second stage of phagocytosis [41]. The precise signalling molecules involved depend on the exact receptor, but there are always numerous different components (including kinases, adaptor proteins, small GTPases and lipid modifiers) linked together in complex interacting networks consisting of both activating and inhibitory components [42]. Various events follow initial receptor-ligand bonds and receptor signalling, such as receptor clustering at the site of engulfment. This allows new receptor-ligand bonds to form so that the cell membrane can gradually extend around the target, creating what is called the phagocytic cup (Fig. 1). It is worth noting that there is debate as to whether some forms of phagocytosis, such as complement-mediated phagocytosis, involve the target "sinking" into the cell (as in clathrin-mediated endocytosis) rather than outward-extending pseudopods [43-48]. The gradual membrane wrapping is often described as zipper-like, with receptor engagement needed around the entire target for successful internalisation [49-51].

Another important event is rearrangement of the cytoskeleton. The cytoskeleton is a dynamic network of filaments within the cell, made from various different proteins, that plays a role in giving shape to the cell, force generation, and intracellular movement of material. These filaments include actin and microtubules, both of which play a role during phagocytosis [52,53]. For example, actin is recruited to the phagocytic cup and is likely to contribute to the force that pushes the membrane around the target [18]. Further, without actin, the success of phagocytosis is severely diminished, especially for large target particles [51]. Actin may even have a role in receptor clustering at the phagocytic cup [54]. Finally, elements of the cytoskeleton have their associated motor proteins that walk along them and can generate forces. Of particular importance for phagocytosis are myosins (motor proteins for actin) that play an important role in closing the phagocytic cup towards the end of engulfment [17].

As the phagocytic cup progresses around the target, the membrane must both change shape and continue to envelop the target. This requires modifying the membrane composition by, for example, altering the lipid composition [55]. Spare membrane, in the form of membrane wrinkles, can initially be used to extend around the target. However, when (or if) this spare membrane is exhausted, new membrane must be created or brought from internal stores before engulfment can continue [56-58]. The importance of the cell membrane during engulfment means that its various biophysical properties, such as tension and bending modulus, play a critical role in determining if 
phagocytosis can successfully complete.

The third stage of phagocytosis involves closing the membrane at the top/end of the target particle (Fig. 1) to form the phagosome. This requires a change in membrane topology so that the particle moves from the outside to inside the cell. This fascinating process, which bears some similarities to cytokinesis (the process of physically dividing the cell during cell division), is not yet fully understood, either experimentally or theoretically.

Once the phagosome is formed, the final stage of phagocytosis starts with the gradual change of the phagosome membrane composition to form the mature phagosome. This can then fuse with lysosomes, a type of organelle that contains a multitude of digestive enzymes, with the result that the phagosome becomes highly acidic. Finally, the digestive enzymes try to degrade the target which, if successful, marks the end of phagocytosis.

\subsection{Phagocytosis in the immune system}

Our immune system is made of two components, the innate immune system, which provides an immediate non-specific response to infection, and the adaptive immune system, which is slower, antigen-specific and provides memory of past infections. Phagocytosis is a critical component of both systems. Many different organs, tissues and cells are involved in immune response, including the spleen, thymus, lymph nodes and tonsils [59].

Specialised white blood cells, called professional phagocytes, are able to carry out phagocytosis within the immune system. These include neutrophils, macrophages, mast cells and dendritic cells. During infection, these cells migrate via chemotaxis to the site of infection. Once there, after phagocytosing a foreign particle, they can present antigens derived from the ingested particle to other cells of the immune system (the lymphocytes), thus providing the memory component of the adaptive immune system [42] and initiating an inflammatory response [60]. Various other cells, called non-professional phagocytes, can also perform phagocytosis. These include epithelial cells, fibroblasts and endothelial cells. However, their lack of relevant receptors means that these cells cannot engulf the same range of targets as professional phagocytes [61].

Phagocytosis research often focuses on neutrophils and macrophages. Neutrophils are the most abundant type of professional phagocyte, accounting for over half of all white blood cells $[62,63]$. They are a type of granulocyte, meaning that they contain many intracellular granules (tiny vesicles consisting of enzymes to destroy ingested matter) within each cell. Typically they have segmented, multi-lobed nuclei. They are involved in the early stages of infection and normally live for only about 24-48 hours. After 
phagocytosis, they can stimulate the appearance of macrophages, which in turn recruit more neutrophils, leading to a co-ordinated immune response. Macrophages, on the other hand, are mononuclear cells that arise when monocytes differentiate within tissues $[64,65]$. They are slower to respond than neutrophils and play a role in removing (via phagocytosis) dying neutrophils. Macrophages have multiple specialised types in various tissues, such as Kupffer cells in the liver, microglia in the brain and osteoclasts in bone. In addition, they can adopt at least two forms: M1 macrophages (often called "killer" macrophages) that encourage inflammation and M2 macrophages (known as "repair" macrophages) that suppress inflammation and promote tissue repair.

\subsection{Physics, mathematics and modelling}

In the last fifty years, theoretical approaches to biology and medicine have gradually become more and more powerful and accepted, with contributions in almost every related discipline, from evolution to protein structure and from epidemiology to membrane mechanics [66-70]. These theoretical approaches include areas of physics (such as thermodynamics, fluid dynamics and soft matter), computing (such as image analysis, bioinformatics and numerical simulation) and mathematics (such as differential equations, dynamical systems and network theory). Notable successes, amongst many, have included how bacteria move during chemotaxis [71,72] and the HodgkinHuxley model describing action potentials in neurons [73].

Unfortunately, the word "model" has multiple different meanings in biology, including model organisms, animal models of disease, protein network models, statistical models, and mathematical models. This has (and continues) to cause enormous confusion, especially when physicists, mathematicians, biologists and clinicians interact. Here, by "model", we are always referring to mathematical models, often with input and motivation based on physical concepts. These include ideas like reaction-diffusion equations, membrane mechanics and spatio-temporal dynamics.

In turn, mathematical modelling of biological processes can be subdivided in various broad areas, such as phenomenological models, all-inclusive models (such as the Human Brain Project), and simplified mechanistic models. Although all approaches have their uses, it is in our opinion often the simplest models that lead to the best progress. This is because the simplest models, which purposely try to include only a few key components, have the fewest parameters (which always need to be measured, estimated or fit) and so tend to generate the greatest number of testable predictions and the greatest understanding. Of course, models that are too simple cannot hope 
to capture the huge complexity of real biological systems. Often the optimal involves finding the simplest useful model, and so maximising the chance of getting "more out than we put in", which we think should always be one of the most important yardsticks by which the success of theoretical approaches is measured.

The complexity of phagocytosis may explain the scarcity of theoretical approaches to such an important topic. This is also true, although to a lesser extent, of other forms of endocytosis. In our opinion this is due to the mistaken belief that every component of the process, including remodelling of the membrane, the cytoskeleton, intracellular signalling, and the influence of neighbouring cells, must be included. However, recent work has made progress by utilising simplified models that include only some of these ingredients and make various idealising assumptions. These includes models that focus on the motion of receptors, models that examine the fundamental types of forces involved, and models that determine the membrane shape by minimising some energy functional. That useful understanding and verifiable predictions are still generated, justifies these approaches.

\section{Receptor models}

Unlike some other types of endocytosis, phagocytosis needs receptors in the cell membrane to interact with some ligand on the target. This requires the receptors to be present at the edge of the phagocytic cup in order to bind with unbound ligands and so facilitate further membrane wrapping of the target. How this is achieved is still poorly understood and naturally lends itself to biophysical and mathematical models of receptor motion, including effects such as receptor diffusion and more active types of motion. In this section, after a brief introduction to the role of receptors in phagocytosis, we discuss receptor models of engulfment, first in receptor-mediated endocytosis and then in phagocytosis.

\subsection{Phagocytic receptors}

Receptors can bind targets either directly or via opsonins (such as antibodies and complement proteins). This connection is often assumed to be irreversible, so that the membrane gradually wraps the target in a ratchetlike manner [51]. In particular, this assumption simplifies various modelling approaches.

Receptors that directly bind targets, often called pattern-recognition receptors, do so by recognising some component of the target that is not found 
in the organism itself. These components, referred to as pathogen-associated molecular patterns (PAMPs), include bacterial proteins such as flagellin (the main constituent of flagella), double-stranded RNA (associated with viruses), and fungal particles. Examples of such receptors are Dectin-1, which recognises various plant and fungal glucans, and the mannose receptor, which identifies a sugar often found on pathogen surfaces [42].

Opsonic receptors bind to proteins that have been attached to target particles leading to improved recognition and binding. These include the complement and Fc receptors. For example, the Fc $\gamma$ receptor (sometimes abbreviated $\mathrm{Fc} \gamma \mathrm{R}$ ), which we will often focus on here, recognises IgG, the most common type of antibody [74]. Finally, there are various apoptotic receptors that engage with apoptotic cells that are displaying "eat-me" signals. These include the PS receptors, which identify the lipid phosphatidylserine (PS), and the CD36 scavenger receptor [38].

\subsection{Receptor models in endocytosis}

A consequence of phagocytic models that focus chiefly on receptors is that they also often apply to other forms of receptor-mediated endocytosis (such as clathrin-mediated endocytosis). In fact, in some cases, the phagocytic models originated as models for other types of endocytosis.

Although the membrane is two-dimensional, many receptor models assume circular symmetry when the target is viewed directly from above (looking straight down onto the cell). Of course, this implies that the target itself must be circularly symmetric, so that many models only focus on spherical targets. Recent extensions to non-spherical cases will be discussed in $\S 5$. This means that the system becomes effectively one-dimensional and can be parameterised by $r$, the distance from the centre of the cup (i.e. the point where the cell first makes contact with the target). This often simplifies both the analytic and numeric analysis. Note that in this review the number of dimensions refers to the number of spatial coordinates in the model, not to the number of finite dimensions of the target shape as in some other work.

The two key variables in receptor models are the density of receptors, $\rho(r, t)$, and the size of the cup, $a(t)$ (Fig. 2A). The receptor density is a function of both the distance from the cup $r$ and the time $t$, whereas cup size depends only on $t$. Since the receptors are defined by a density, there is an implicit assumption that there are enough receptors for such a description to make sense. The cup size must be defined carefully since the cup is not flat. Normally, $a(t)$ measures the arc distance along the membrane from the centre of the cup $(r=0)$ to the edge. Particular models often include addition variables, such as the actin density, but $\rho(r, t)$ and $a(t)$ are ubiquitous in 

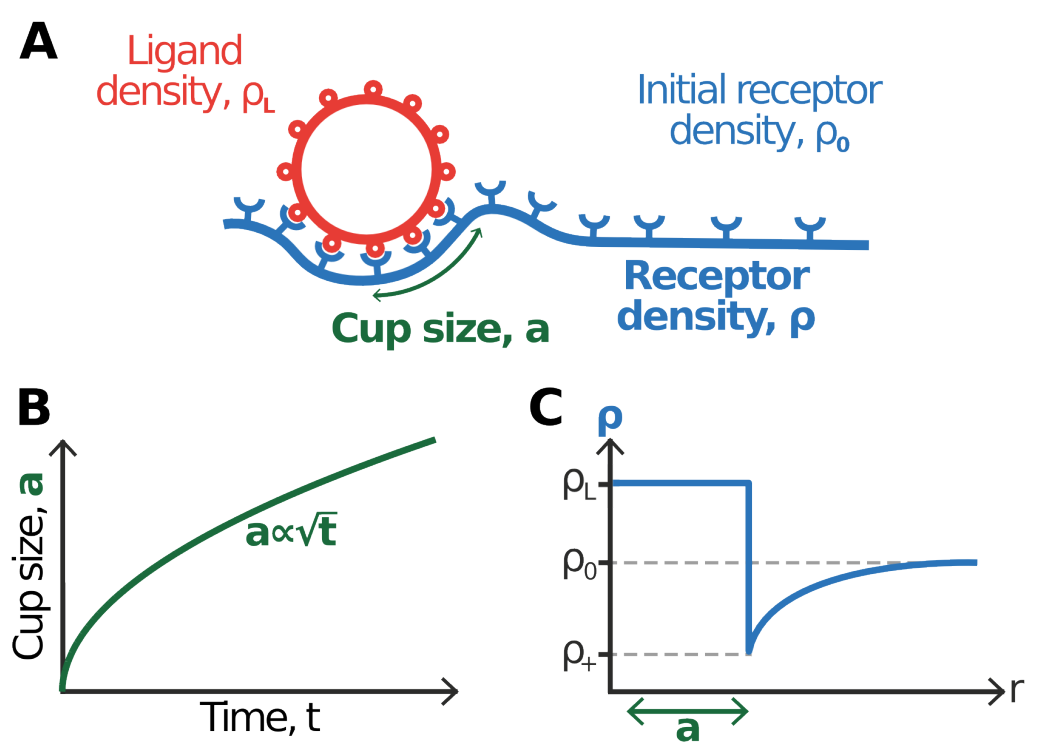

Figure 2. The basic receptor model. (A) Schematic of the model showing the cup size $a$, receptor density $\rho$, initial receptor density $\rho_{0}$ and ligand density $\rho_{L}$. Receptors at the edge of the cup bind unattached ligands on the target and so gradually increase the cup size. (B) Sketch of how the cup grows in size in the pure diffusion model $(a(t) \propto \sqrt{t})$, with the engulfment rate progressively slowing down during engulfment. (C) Sketch of the receptor density profile showing that, outside the cup, the density gradually rises with increasing distance from the cup. This leads to an inward receptor flux and so to cup growth.

receptor models.

It is usually assumed that, before endocytosis or phagocytosis begins, there is no cup $(a(0)=0)$ and a constant receptor density $\rho_{0}$ throughout the membrane $\left(\rho(r, 0)=\rho_{0}\right)$. In the simplest models, it is also assumed that the receptor density within the cup matches the ligand density $\rho_{L}$ on the target, i.e. it is assumed that, within the bound region, all ligands are bound to receptors with no unbound receptors left over. This implies that, at all times, $\rho(r, t)=\rho_{L}$ for $r<a(t)$. Further, a boundary condition at large $r$ is needed, which is often taken as $\rho(\infty, t)=\rho_{0}$.

One of the most important unsolved problems in phagocytosis is to determine which factors govern receptor motion within the membrane, and whether this motion is passive, active or a mixture of the two. In one of the earliest receptor models of endocytosis by Gao et al., receptors are assumed to simply diffuse freely within the unbound part of the membrane $[75,76]$. 
This means that the receptor density $\rho$ for $r>a(t)$ is described by

$$
\frac{\partial \rho}{\partial t}=\frac{D}{r} \frac{\partial}{\partial r}\left(r \frac{\partial \rho}{\partial r}\right)
$$

where $D$ is the receptor diffusion constant. This is simply the radial part of the two-dimensional diffusion equation. (The angular part only becomes important for certain non-spherical particles as we discuss in §5.) For simplicity, contributions from the fact that receptors must diffuse on a curved surface are ignored. As we describe below, some later models rectify this.

The equation for the cup size $a(t)$ can be derived by conserving receptor number at the edge of the cup: all receptors entering the cup are assumed to bind free ligands (at density $\rho_{L}$ ) and hence increase the cup size. This leads to

$$
\frac{d a}{d t}=\frac{D \rho_{+}^{\prime}}{\rho_{L}-\rho_{+}},
$$

where $\rho_{+}=\rho(a(t), t)$ is the receptor density at the cup edge, the numerator is the inward receptor flux, and the denominator accounts for the increase in density from $\rho_{+}$to $\rho_{L}$ as receptors enter the cup. The ratchet mechanism implies that $a(t)$ can never decrease, so that outward receptor fluxes are assumed to correspond to $\dot{a}=0$.

These two equations together model the receptor density profile and cup growth. Interestingly, they are identical to the Stefan problem, which is the classic physics problem describing phase transitions such as the melting of a solid [77-79]. For example, when applied to a melting ice cube, $\rho$ and $a$ are interpreted as the temperature and ice cube size respectively. In fact, to be more precise, these equations are actually the supercooled Stefan problem which describes, for example, the freezing of supercooled water. Thus, the process of endocytosis can be viewed as a phase transition between bound and unbound receptors.

In order to find a unique solution, one extra boundary condition must be imposed. There are multiple ways that this can be found and motivated. One possibility is to require that energy is conserved as receptors cross the cup boundary. In this case, it is important to first decide which effects, such as membrane curvature, membrane tension and receptor-ligand binding, should be included in the expression for the energy. In fact, different models disagree on this due to different estimates of the relative importance of each contribution. The approach taken by Gao et al. is to use the free energy with, in addition to the entropy term, contributions from membrane curvature and receptor-ligand binding. In this case, the extra boundary condition is found to be a condition on $\rho_{+}$(the receptor density at the edge of the cup) and is 
given by

$$
\frac{\rho_{+}}{\rho_{L}}-\ln \left(\frac{\rho_{+}}{\rho_{L}}\right)=\mathcal{E}-\frac{2 \mathcal{B}}{\rho_{L} R^{2}}+1
$$

where $\mathcal{E}$ is the binding energy per receptor-ligand bond, $\mathcal{B}$ is the membrane bending modulus, and $R$ is the target radius. It is worth noting that, since we are here considering a spherical target, this expression does not involve time so that the receptor density at the cup edge is always the same, even as the cup grows.

One advantage of this model is that, for spherical target particles and $\rho_{0}<\rho_{L}$, an analytic solution exists and is given by

$$
\begin{aligned}
\rho(r, t) & = \begin{cases}\rho_{L} & \text { for } r<a \\
\rho_{0}-\left(\rho_{0}-\rho_{+}\right) \frac{E_{1}\left(r^{2} / 4 D t\right)}{E_{1}\left(\alpha^{2}\right)} & \text { for } r \geq a\end{cases} \\
a(t) & =2 \alpha \sqrt{D t},
\end{aligned}
$$

where $E_{1}(x)=\int_{x}^{\infty} \frac{e^{-u}}{u} d u$ is the exponential integral and $\alpha$ is found by solving

$$
\alpha^{2} e^{\alpha^{2}} E_{1}\left(\alpha^{2}\right)=\frac{\rho_{0}-\rho_{+}}{\rho_{L}-\rho_{+}}
$$

Since the receptor density at the cup edge is independent of time, $\alpha$ is also constant throughout the whole engulfment process. Later, when nonspherical targets are considered, this will no longer be the case. Three points are worth noting about this solution. Firstly, outside the cup, the receptor density gradually rises with increasing $r$, tending towards $\rho_{0}$ as $r \rightarrow \infty$ (Fig. 2C). This results in an inward receptor flow and so to progressive wrapping of the target. Secondly, the cup grows with the square-root of time, $a \propto \sqrt{t}$, which is characteristic of Stefan problems and of diffusion problems in general (Fig. 2B). Thirdly, the total engulfment time, i.e. the time until $a=\pi R$, can be found from $t=\pi^{2} R^{2} / 4 D \alpha^{2}$. (Of course, this does not imply that the engulfment time scales simply like $R^{2}$ since $\alpha$ also depends on $R$ via $\rho_{+}$.)

\subsection{Receptor models in phagocytosis}

At first glance, this model of Gao et al. for endocytosis seems unlikely to apply to phagocytosis as well: phagocytosis is a much more active process, with the cytoskeleton pushing the membrane around the target particle (Fig. 1). However, various modelling approaches have suggested that this is not the case and that this model can be adapted to describe phagocytosis $[80,81]$. 
These approaches typically argue that the role and motion of receptors is similar in all forms of receptor-mediated endocytosis. For example, that receptors must move around the two-dimensional membrane and be present at the cup in order for engulfment to proceed is not unique to phagocytosis. This suggests that the relevant biophysical processes, such as diffusion, are likely to be applicable to all types of endocytosis [80].

However, it is important to realise that phagocytosis does have important differences to other forms of endocytosis and that failure to capture these differences is unlikely to lead to successful models. Firstly, outward membrane protrusions that form the phagocytic cup must be more curved than internal invaginations. This is because they must bend back on themselves in order to rejoin with the rest of the membrane [80]. This extra membrane curvature must be taken into account. Secondly, in addition to passive diffusion, other types of receptor motion, such as active drift, are likely to play an important role. Thirdly, the cytoskeleton, in particular actin, cannot be neglected. Although actin is involved in many types of endocytosis [82-84], its role seems most critical in phagocytosis. In fact, engulfment (especially of large particles) is largely abolished when actin is depolymerised [85, 86]. Fourthly, the effect of signalling must be carefully considered and can no longer be ignored. As with actin, the added difficulty of engulfing large particles means that signalling during phagocytosis is likely to play a more pronounced role than in other forms of endocytosis, and is probably necessary to explain certain features, such as the sharp switch in phagocytic engulfment rate around half engulfment [80].

One of the first models to examine receptor motion during phagocytosis is the elegant work by van Zon et al., which studies the possible ultimate fates of phagocytosis, focusing on how and where engulfment stalls when phagocytosis fails [87]. There are various important novelties in this work that are introduced to capture the nature of phagocytosis. These include taking into account that, unlike the rest of the membrane, diffusion within the cup cannot necessarily be approximated as diffusion on a flat surface (extra curvature terms are required in Eq. (1)), and arguing that receptor-ligand binding energy is a relatively small contribution to the total energy and so can be ignored. Further, in addition to receptors outside the cup, they also consider the density of both bound and unbound receptors within the cup region. This allows the density of bound receptors to vary around the target and captures the fact that not all receptors within the cup need be bound. Finally, the cytoskeleton is included by allowing bound receptors to recruit actin to the cup. Thus, in this model, the role of bound receptors is to both stimulate accumulation of actin and determine the cup shape. Although actin is only included as a macroscopically-averaged density, ignoring its mi- 
croscopic network structure, this is an important first attempt to model the function of actin during phagocytosis. Ultimately, the shape of the phagocytic cup in this model is generated by two forces: a pushing-out force due to actin (proportional to the local concentration of F-actin) and a restoring force due to the cell resisting changes to its shape (found by minimising the membrane curvature energy).

Interestingly, this model can explain the large variation in progression and outcome of phagocytosis that is observed even for identical particles. In particular, it has been shown (experimentally) that engulfment tends to either stall before half-way or reach completion, thus leading to a bimodal distribution for cup sizes at late times [87]. This is neatly explained in the model by van Zon et al. as a mechanical bottleneck: the point where the cell must exert most force to further extend the cup occurs at half engulfment, where the edge of the cup is at its widest. So if a cell can generate sufficient force to reach half engulfment, it should also be able to generate enough force to complete engulfment. Finally, van Zon et al. were able to predict and then confirm experimentally that reducing actin does not necessarily lead to stalled cups [87].

Another approach to extending the Gao et al. model to phagocytosis involves adding active receptor motion [80]. Such active motion implies that receptors are directly clustered at the phagocytic cup by, for example, coupling receptors to the actin cytoskeleton as happens in immunological synapses [88]. The simplest way that this can be included in a model is to replace receptor diffusion by a constant inward drift with speed $v$ (the drift velocity). Then Eqs. (1) and (2) for $\rho(r, t)$ and $a(t)$ become

$$
\begin{aligned}
& \frac{\partial \rho}{\partial t}=\frac{v}{r} \frac{\partial}{\partial r}(r \rho), \\
& \frac{d a}{d t}=\frac{v \rho_{+}}{\rho_{L}-\rho_{+}},
\end{aligned}
$$

with the same initial conditions as in the diffusive model (Fig. 3A). Since the $\rho$-equation is now first order in time, there is no longer any need for an extra boundary condition (found above by conserving free energy), and the analytic solution becomes

$$
\begin{aligned}
\rho(r \geq a, t) & =\rho_{0}\left(1+\frac{v t}{r}\right), \\
a(t) & =\frac{1}{\sqrt{\rho_{L} / \rho_{0}}-1} v t .
\end{aligned}
$$

Notably, cup growth is now linear in time. This implies that the time needed 

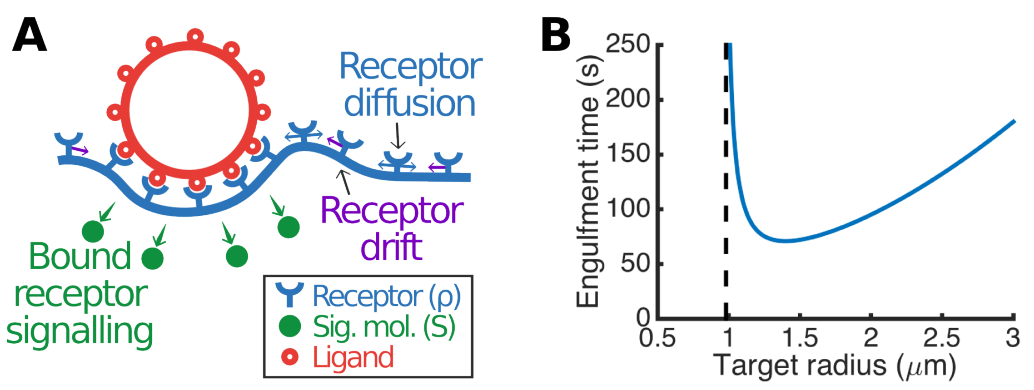

Figure 3. Extensions to the basic receptor model. (A) Addition of receptor drift and a signalling molecule $S$ allows both more complex receptor motion and the recruitment of actin to the phagocytic cup by bound receptors. (B) The total engulfment time against target radius, showing an optimal radius for the quickest possible engulfment.

for complete engulfment, $\pi R\left(\sqrt{\rho_{L} / \rho_{0}}-1\right) / v$, grows linearly with target radius.

Of course receptor motion is unlikely to be either pure diffusion or pure drift, but some combination of the two. Such models must be solved numerically and typically result in cup growth that is initially diffusive $(a(t) \sim \sqrt{t})$ and later dominated by drift $(a(t) \sim t)$ [80]. Although $v$ has so far been assumed to be constant, it is quite possible that, in real cells, any drift will depend on $r$, with larger drift occurring nearer the cup. Future models are needed to investigate this possibility.

\subsection{The effect of signalling}

There are a multitude of signalling molecules involved in phagocytosis, which are activated and/or recruited when receptors bind ligands [42,89-91]. Determining the role for each of these is an important area of ongoing research. A first step towards incorporating signalling in the mathematical models involves adding a single signalling species to the above receptor models [80]. Although this may seem a simplification too far, it is sufficient to capture the essential nature of signalling and its effect on receptor dynamics. Later models will be able to add additional signalling species and their internal interactions. By assuming that the signalling molecule only moves within the membrane (as is the case for some small GTPases), its density can be represented as $S(r, t)$. Again, this is an assumption that can be relaxed in future models, but still permits progress to be made. Allowing $S$ to diffuse with diffusion constant $D_{S}$ (which is generically different to the receptor diffusion 
constant), degrade (with lifetime $\tau$ ) and be produced by bound receptors (with rate $\beta \rho_{L}$ ) leads to

$$
\frac{\partial S}{\partial t}=\frac{D_{S}}{r} \frac{\partial}{\partial r}\left(r \frac{\partial S}{\partial r}\right)+\beta \rho_{L} \Theta(a-r)-\tau^{-1} S
$$

where the Heaviside function ensures that $S$ is only produced within the cup region (Fig. 3A).

The final step in models of this type is to couple signalling to receptor motion. There are a number of ways this can be achieved, including $S$ affecting the receptor directly (via, for example, altering the receptor diffusion constant, producing new unbound receptors or modifying receptor-ligand binding) or by changing properties of the membrane (such as the bending modulus or the availability of spare membrane). One particularly interesting approach is to allow the signalling molecule to affect the receptor drift velocity, so that drift (which is assumed absent at the start of phagocytosis) is turned on when engulfment reaches a certain point [80]. This leads to multiple stages of engulfment characterised by different engulfment rates, a topic that we will return to in $\S 4$.

With the basic receptor model in place (including receptor diffusion, receptor drift and signalling) it is possible to predict how the total engulfment time depends on the model parameters, in particular the target size and ligand density [92-97]. In both cases the dependence is nontrivial due to the nonlinear nature of Eqs. (3) and (5). Further, changing the target radius not only affects the engulfment rate via $\alpha$, but also the final area of the phagocytic cup. In both cases there is some intermediate parameter value that leads to the quickest engulfment: larger or smaller values increase the engulfment time or even result in incomplete, stalled phagocytosis. For the target radius this occurs because (i) small targets have large curvature and thus require more tightly curved cups, and (ii) large targets need more membrane to wrap them. Thus, intermediate-sized particles should be phagocytosed quicker than smaller or larger particles [75] (Fig. 3B). Similarly, low ligand density leads to few receptor-ligand bonds and so low levels of signalling, and high ligand density needs a high density of receptors to build up at the cup in order to bind the numerous ligands. Thus, an optimal ligand density for the quickest engulfment is predicted [80]. It is worth noting that this conclusion is based on the assumption that all target ligands must be bound. Future models could relax this assumption and examine how this affects engulfment time. This could then perhaps explain recent results on the effect of ligand density during frustrated phagocytosis of a flat surface [98]. 


\section{$3 \quad$ Force and energy models}

The motion of receptors is just one aspect of phagocytosis. It is also important to understand the forces involved, either directly or via minimisation of some energy functional. For example, the cell must push the membrane around the target particle, overcoming the membrane tension. In particular this outward force must only act at the edge of the target particle: pushing directly at the target base will only result in the target being pushed away from the cell. Progress in this area has benefited from recent dualmicropipette time-lapse experiments that allow the shape of the cup to be visualised at high resolution. In this section, we will first review these experiments, before discussing force-based and energy-based models that try to capture and understand the shape of the phagocytic cup.

\subsection{Dual-micropipette experiments}

Immune cells tend to adhere to surfaces making analysis difficult. Using glass micropipettes to isolate and position both the cell and the target can avoid this. Then direct one-on-one interactions can be observed in isolation of other cells. Further, it is then possible to hand-pick the required cell and target so that, for example, a particular target shape and size can be studied.

Micropipettes are typically used for patch-clamping, microinjection and measurement of mechanical membrane properties. They are made using a micropipette puller, which can produce pipettes with tips from around $0.1 \mu \mathrm{m}$ to $50 \mu \mathrm{m}$ in diameter, and are usually controlled using micromanipulators, which allow accurate submicron positioning. By connecting to a water reservoir, the suction pressure can be adjusted so that cells and targets can be picked up at will $[99,100]$.

The dual-micropipette system involves using two micropipettes, one holding the immune cell and the other the target particle (Fig. 4A) [101]. By lifting both pipettes from the chamber bottom and then releasing the target directly onto the cell surface, it is possible to observe formation of the phagocytic cup and subsequent target motion into the cell. This setup can easily provide high spatial resolution time-lapse images, with sub-second temporal resolution (Fig. 4B). By monitoring the aspiration pressure required to keep a constant length of cell within the pipette, it is also possible to infer the cortical tension at each stage of engulfment [102]. This complements other approaches, such as the use of magnetic targets in oscillating magnetic fields, which allow membrane properties such as stiffness to be measured [103].

There are numerous advantages to the micropipette setup. Perhaps the most notable is the gain in fine control: since both cell and target can be 

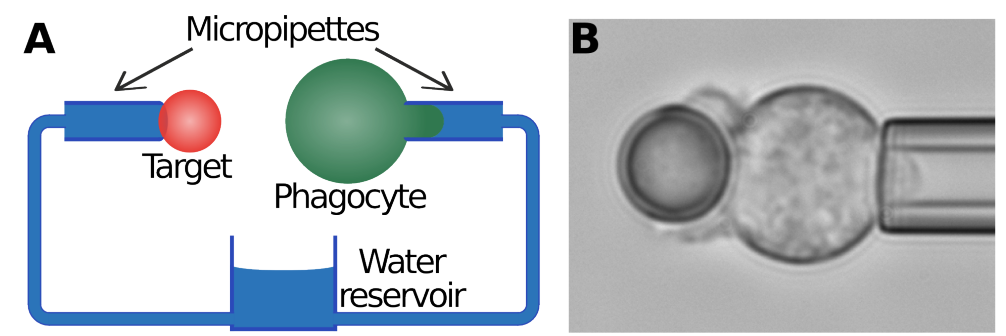

Figure 4. The dual-micropipette system. (A) Schematic of the experimental setup, showing the two micropipettes, the water reservoir, the immune cell and the target. (B) Example image with a $4.6 \mu \mathrm{m}$ polystyrene bead midway through engulfment by a human neutrophil. The second pipette, which initially held the bead, has already been removed. Reproduced from Fig. 2 in Richards et al. (2014) [80]; original image provided by Volkmar Heinrich, UC Davis, California.

selected and manipulated at will, there is no need to wait for chance interaction. In addition, this setup allows the target to be kept in the same focal plane as the cell, so that the phagocytic cup can be viewed from the side. This is in stark contrast to many previous experimental approaches where the target invariably attaches to the upper surface of a flattened, adhered cell, and so makes visualisation of the cup difficult. Further, the use of micropipettes results in approximately spherical cells, which greatly simplifies both image analysis and mathematical modelling. However, it should be noted that, as a consequence, this system differs from in vivo: immune cells within the body tend to both flatten (due to surface adhesion) and interact with other nearby cells. As such, the dual-micropipette setup is most suited for studying fundamental aspects of phagocytosis, in isolation from the rest of the immune system.

\subsection{Force-based models}

Phagocytosis is not a passive process. The cell must use energy to generate the forces that extend the membrane around the edge of the target in a controlled manner. These forces can originate from a variety of sources, including actin polymerisation, cortical tension and cytosol incompressibility. Force-based models focus on determining and understanding these forces, and how they work in combination to engulf foreign particles.

The dual-micropipette setup allows the cortical tension to be measured during engulfment. For example, this was studied in human neutrophils by 
Herant et al., who argued that the cortical tension is responsible for pulling the target inwards [101]. As expected, they found an increase in tension as the cell area gradually increases during phagocytosis, with the extra membrane provided (at least initially) by membrane wrinkles [56,57]. Membrane at later times is likely to be provided by exocytosis of internal vesicles [58]. In addition they discovered that the tension increases only slowly at first, with a much steeper rise later on. They argued that this suggests two classes of membrane wrinkles: ones that easily unravel and ones that are tightly wrapped up. Further, they found that, unlike for large targets, there is not always a noticeable tension increase for smaller targets. Presumably this is because sufficiently small targets need only use spare membrane from the first class of membrane wrinkles. Finally, they examined the difference in tension increase between cells undergoing phagocytosis and cells that are simply aspirated by a micropipette. This showed that the tension remains low for much longer during phagocytosis, which is probably because, once phagocytosis has started, the cell is much more active in providing extra membrane via, for example, unwrapping of wrinkles and fusion of internal vesicles with the cell membrane.

Further work by Herant et al. has directly examined the forces required to account for phagocytic cup shape $[104,105]$. They considered various mechanistic models and compared each to the dual-micropipette data, focusing on matching cell shape, cortical tension and target position. Based on the "reactive interpenetrating flow formalism" of Dembo and Harlow [106, 107], they split the cytoplasm into a mixture of two phases, the cytoskeleton (labelled by ' $n$ ' for network) and the cytosol (labelled by 's' for solvent), and assumed that both phases can be treated as homogenous Newtonian fluids with viscosity dependent on network concentration. By ignoring the microscopic structure, each phase can be represented by a volume fraction, $\theta_{n}(\mathbf{x}, t)$ and $\theta_{s}(\mathbf{x}, t)$, where $\mathbf{x}$ is the spatial position within the cell, $t$ the time and $\theta_{n}+\theta_{s}=1$. In addition, the movement of each phase is described by a velocity field, $\mathbf{v}_{n}(\mathbf{x}, t)$ and $\mathbf{v}_{s}(\mathbf{x}, t)$. Evolution of these four variables is then found by considering incompressibility and conservation of mass and momentum. To model the equilibrium network concentration (which is assumed to be actively modified by the cell during phagocytosis) an additional field $m$ was introduced to describe a "polymerisation messenger". As with the $S$ signalling density defined in some receptor models, $m$ is intended as a proxy for the total effect of intracellular signalling rather than as a particular signalling molecule. This additional field is assumed to diffuse with diffusion constant $D_{m}$, degrade with lifetime $\tau_{m}$ and be produced by the membrane, 


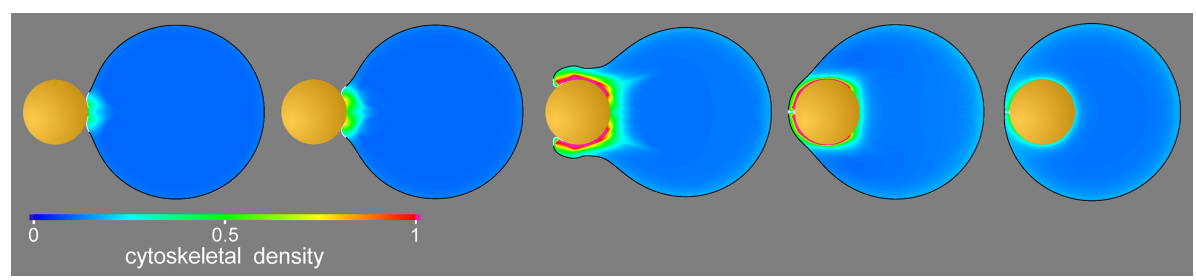

Figure 5. The Herant et al. force-based model. Snapshots from the simulation of a neutrophil engulfing an antibody-coated bead. The colour within the cell represents the actin density. Figure reproduced from Fig. 2 in Herant et al. (2011) [113].

which leads to

$$
\frac{\partial m}{\partial t}=D_{m} \nabla^{2} m-\nabla \cdot\left(m \mathbf{v}_{s}\right)-\tau_{m}^{-1} m,
$$

where the second term describes advection of $m$ by cytosolic flow. The whole system was solved numerically using a finite element method based on a mesh of quadrilaterals [108] (Fig. 5).

Herant et al. then argued that the optimal model for phagocytic engulfment requires the interplay of two different forces. First, a repulsive force between the cytoskeleton and the membrane that acts near the edge of the cup and causes the cup to extend around the target. This is likely caused by actin polymerisation at the cup edge. Second, a flattening force that pulls the cup into a thin shell. This was implemented as an additional force $f \propto \exp (-d)$, where $d$ is the distance to the target. Plausibly this is the result of motors (such as unconventional myosins) that are anchored to the bound part of the membrane and pull down the actin cytoskeleton. Herant et al. further showed that other simple models, such as cytoskeletal swelling (network-network repulsion), cannot explain the observed data. Despite the impressive cup shapes obtained from this model (Fig. 5), it is worth noting that it was unable to capture correctly the behaviour of the cortical tension for all bead sizes. This suggests that further work of this type is needed before the forces at play are fully understood.

A mathematical model is normally more convincing if it can easily be adapted to other, similar systems by changing only a few ingredients or parameters. The converse of this, of course, is that it is always important to be wary of purely reactive modelling where each new experimental dataset can only be explained by adding a new part to the model. To this end, Herant et al. investigated whether their model could also capture phagocytosis of zymosan particles. Zymosan is an insoluble polysaccharide component of yeast cell walls that has been used as a model of fungal infection for many 
years $[109,110]$. It has be shown that zymosan phagocytosis differs from that of antibody-coated beads in several ways, most notably by the existence of an initial "push-out" stage $[111,112]$. This stage, which is largely absent for antibody-coated targets, is caused by the membrane pushing directly against the base of the target and so forming a pedestal structure that gradually extends outwards to about $1 \mu \mathrm{m}$ in length. After this, the particle is progressively enveloped and brought inwards as in other types of phagocytosis. As a consequence, the total engulfment time of zymosan is significantly longer than for other targets with, for example, $3 \mu \mathrm{m}$ zymosan particles taking around 2.5 times longer than antibody-coated beads of the same size [111].

Herant et al. found that their force-based model could capture zymosan phagocytosis by altering the interaction between the membrane and the cytoskeleton [113]. In particular, the attractive force between the cytoskeleton and target-bound regions of the membrane must be replaced with a protrusive force that pushes the target away from the cell. Thus, with zymosan particles, the membrane pushes on all parts of the target rather than just at the cup edge. Herant et al. speculate that this is related to the protein complexes that link bound receptors and the cytoskeleton, with antibody receptors associated with stronger linkage and so with greater suppression of cell protrusion [113]. It remains to be seen whether a more unified force-based model can be developed that captures phagocytosis of both antibody-coated beads and zymosan particles with the same forces.

The fundamental nature of this type of modelling, with focus on only a few key biophysical forces, suggests that it should be readily extensible to other similar systems. For example, it has traditionally been assumed that phagocytosis is distinct to chemotaxis (the directed movement of cells towards chemical stimuli). However, recent work (again using the dual-micropipette system) argues that the difference for immune cells may be far less than previously thought [31]. In both phagocytosis and chemotaxis, receptors bind some ligand (either on the target for phagocytosis or freely diffusing for chemotaxis), which initiates a signalling cascade, causing recruitment of actin and outwards deformation of the cell membrane (either around the target for phagocytosis or towards the source for chemotaxis). Future work should examine this link by ascertaining whether the above force-based models can (perhaps with minor changes) capture both phagocytosis and chemotaxis. In addition, phagocytosis of additional target types (other than antibody-coated beads and zymosan particles) is likely to be covered by similar models. For example, some species of the fungus Coccidioides (such as Coccidioides immitis and Coccidioides posadasii which cause Valley fever) can avoid detection by the immune system, even though they are phagocytosed when placed in 
direct contact with neutrophils [114]. Understanding the intracellular forces at play during Coccidioides phagocytosis may well shed light on this.

\subsection{Energy-based models}

The bending energy of a membrane plays an important role in determining its shape. This is especially important during phagocytosis, where the curvature around the target and at the cup base can be relatively high. In pioneering work in the 1970s, Helfrich proposed that the total bending energy of a lipid bilayer is given by $[115,116]$

$$
E_{\text {curv }}=\int d S\left(\frac{k}{2}\left(H-H_{0}\right)^{2}+\tilde{k} K\right)
$$

where the integral is taken over the entire membrane surface, and $k$ and $\tilde{k}$ are bending rigidities. Here $H$ and $K$ are the mean and Gaussian curvatures respectively, which are given in terms of the two principal curvatures, $\kappa_{1}$ and $\kappa_{2}$, as $H=\frac{1}{2}\left(\kappa_{1}+\kappa_{2}\right)$ and $K=\kappa_{1} \kappa_{2}$. The spontaneous curvature, $H_{0}$, accounts for any innate curvature due to the membrane's lipid and protein structure. For more details, see the reviews by Lipowsky and Seifert [117119].

The Helfrich energy has often been used to model the shape of the cup during non-phagocytic types of endocytosis, such as clathrin-mediated endocytosis [120-122]. This normally involves adding various other terms to the total energy, such as a tension term and/or a negative energy contribution from receptor-ligand bonds. Further, various other improvements have been considered, such as including slippage between lipid layers [123-125] and adding a role for the cytoskeleton [126].

This formalism has also been adapted to apply to phagocytosis. In particular, Tollis et al., with various improvements, used the Helfrich energy to examine the role of stochasticity and actin during phagocytosis [51]. They based their model on a ratchet mechanism, where receptor-ligand bonds are never broken and so targets can never be unwrapped. They argued that one of the main roles for actin during phagocytosis is to implement this ratchet mechanism: when a membrane fluctuation causes cup growth, the actin network extends into the gap and so prevents unwrapping [127]. However, it is important to point out that, although this demonstrates the importance of stochasticity, it is likely to oversimplify the role played by actin [105].

The model of Tollis et al. describes the membrane as a two-dimensional surface embedded in three dimensions, with thermal fluctuations (the stochastic element) gradually pushing the membrane around the target particle. By 
assuming that the Gaussian curvature and spontaneous curvature can be ignored, the total energy is taken to be

$$
E_{\text {total }}=\int d S\left(\frac{k}{2} H^{2}+\sigma+V_{\mathrm{LR}}\right)+\bar{k}\left(V-V_{0}\right)^{2},
$$

where $\sigma$ is the surface tension. The $V_{\mathrm{LR}}$ term describes receptor-ligand binding and is taken as a step function: $V_{\mathrm{LR}}=-V_{\mathrm{LR}}^{0}$ (a constant) within some fixed distance of the target and zero otherwise. Finally, the last term is a force that tries to conserve the cell volume $V$, with $V_{0}$ the initial cell volume. Simulations were performed using a Monte Carlo method based on a finiteelement approximation of the cell membrane: fluctuations that decrease the membrane energy are always chosen, whereas those that increase $E_{\text {total }}$ are accepted with some non-zero probability.

Tollis et al. first examined how the cup shape depends on their parameter values, showing that thin phagocytic cups require both low surface tension (small $\sigma$ ) and a tight cell-volume constraint (large $\bar{k}$ ). They then studied the role of actin by comparing an active zipper model (receptor-ligand bonds are permanent) with a passive model (receptors can unbind due to lack of actin polymerisation), finding that, even without actin, phagocytosis (at least for small targets) still occurs, although at a slower rate and with more variation in cup shape. Finally, they confirmed their predictions using fibroblast-like COS-7 cells transfected with the Fc $\gamma$ receptor, both by comparing the wildtype receptor to a signalling-dead mutant and by treating with cytochalasin $\mathrm{D}$ (an inhibitor of actin polymerisation).

\section{Stages of engulfment}

One of the chief advantages of the dual-micropipette system is that the detailed shape of the phagocytic cup can easily be examined as a function of time. For example, it can be used to determine how the size of the cup, normally defined as the arc length from the cup centre and labelled by $a$, changes in time from zero to full engulfment. In particular, as we now explain, it has been shown that the rate of cup growth (i.e. $\frac{d a}{d t}$ ) is not constant but shows distinct stages with each characterised by different receptor dynamics.

An example frame from this kind of data is shown in Fig. 4B, where a $4.6 \mu \mathrm{m}$ polystyrene bead is about to be engulfed by a human neutrophil. Since the bead is coated in IgG antibody, it will be recognised and bound by Fc $\gamma$ receptors within the membrane of the immune cell. The high quality of this kind of image means that it can easily be interpreted by automatic image analysis software. For example, using a combination of edge detection, 

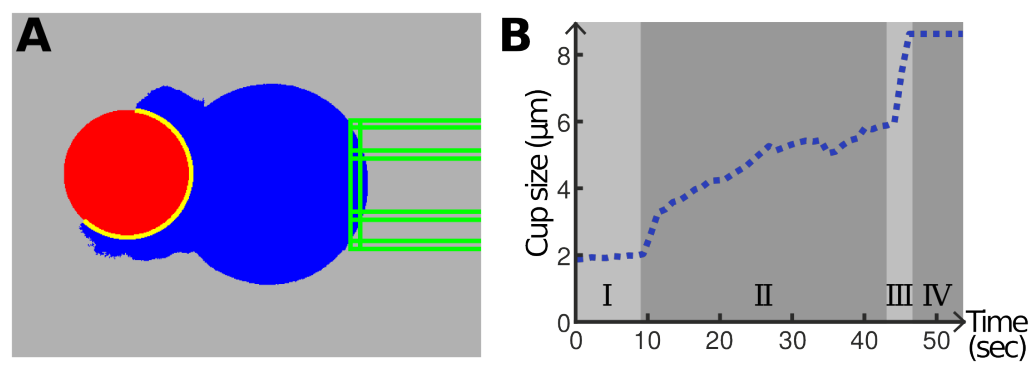

Figure 6. The multistage nature of engulfment. Example of a real human neutrophil challenged with a $4.6 \mu \mathrm{m}$ spherical bead. (A) Image analysis of the same frame shown in Fig. 4B, with the cell in blue, the bead in red, the pipette in green and the phagocytic cup in yellow. (B) Growth of the cup in time for the same single cell and bead showing (I) the initial adherent period $(0-9 \mathrm{~s})$, (II) the first engulfment stage with relatively slow engulfment rate (9-44s), (III) the much quicker second engulfment stage (44-46s), and (IV) the period after engulfment finishes $(>46 \mathrm{~s})$. The cup size $a$ has been averaged over the upper and lower lobes. Reproduced from Fig. 2 in Richards et al. (2014) [80].

thresholding and Hough transforms (a way of identifying patterns such as lines and circles), it is possible to accurately extract both the position of the bead and the shape of the phagocytic cup (Fig. 6A) [80].

It is then possible to determine how the cup grows in time, which recent work has shown can be divided into a number of different regimes (Fig. 6B). Initially, there is a period when the bead adheres to the cell but phagocytosis has not started [101]. At present it is not clear whether this is a general feature of phagocytosis or simply an artefact of the dual-micropipette system. However, once engulfment begins, there is a clear split into two distinct stages. The first is characterised by relatively slow engulfment, which seems to get progressively slower and slower, whereas the second is much quicker and takes only a few seconds [80]. In fact, for beads around $5 \mu \mathrm{m}$ in diameter, the first stage takes over three times as long as the second. Interestingly, the crossover between the two stages always seems to occur when the target is about half engulfed, independent of the particle size. Although this slowthen-fast behaviour is the opposite of that found by Masters et al. [128], this is likely because they only considered spreading on a flat surface (ignoring any target curvature) and involved a large initial contact area (probably missing the initial slow stage).

It is possible to capture this multi-stage behaviour using a receptor model 
that includes receptor diffusion, receptor drift and signalling [80]. This model suggests that the initial, relatively slow stage of engulfment is the result of passive receptor diffusion: new receptor-ligand bonds are only formed if a receptor happens to diffuse to the edge of the phagocytic cup. This is in sharp contrast to the second stage which requires, in addition to receptor diffusion, both receptor drift and signalling. This makes the second stage much more active and probably requires the cell to expend energy. The exact cause of this active behaviour is not yet known, but could involve coupling receptors to the cytoskeleton or modification of the membrane near the cup edge. Interestingly, this model could also provide an alternative explanation for why phagocytosis normally either stalls before half-way or reaches full engulfment [87].

It is tempting to speculate on the evolutionary origin of this two-stage nature of phagocytosis. It is possible that there was originally only one stage governed by passive receptor diffusion, making this "proto-phagocytosis" more similar to other forms of endocytosis. However, this would result in slow engulfment times and hamper engulfment of large particles, suggesting that the second active stage could have evolved later in order to improve the speed and efficiency of phagocytosis.

\section{$5 \quad$ Target shape dependence}

As in most work in phagocytosis, both experimental and theoretical, we have so far assumed a spherical target. There are various reasons for this, including the ease of fabrication, ready availability, simplified image analysis, and less-involved modelling. For example, identifying a sphere in an image (via a Hough transform) is simpler and much quicker than for more complex shapes such as ellipsoids. Also, mathematical models involving spheres can normally exploit the target symmetry, resulting in lower-dimensional models that can often be solved by analytic means.

However, real targets can only occasionally be approximated as spheres. Most have completely different shapes. For example, bacteria assume a huge variety of shapes and sizes, ranging from capped-cylinders (such as E. coli and B. subtilis) to filamentous networks (such as Streptomyces), and dividing cells take on an appearance akin to an hourglass. Phagocytes must be able to cope with the full range of shapes that are encountered in the wild.

Understanding how target shape affects phagocytosis is important for a range of biological and medical applications, such as how some bacteria can avoid phagocytosis and how others can invade cells. Further, the usefulness of microparticle drug carriers is often limited by their removal and destruction 
by the immune system: being able to control particle uptake by careful choice of shape and size could lead to improved drug delivery with implications for a whole host of medical conditions [28, 129-133].

In this section, we first cover known experimental results related to target shape and orientation, before discussing models that have addressed this issue. These fall into two broad categories: models that examine highlysymmetric shapes (such as spheroids) and models that can deal with a greater range of targets (such as ellipsoids). Both cases build on the spherical-target models described above, but with extensions that take account of the varying curvature at the edge of the cup as the cell gradually engulfs the target.

\subsection{Experimental results}

In addition to size, it has been known for many years that target shape plays a crucial role during phagocytosis [134]. By examining a range of shapes (including spheres, oblate and prolate ellipsoids, elliptical and rectangular disks, and UFO-shaped particles comprising spheres embedded within oblate spheroids), Champion and Mitragotri argued that the outcome of a cell-target interaction is determined, not by the target size, but by the local target shape at the point of initial contact between cell and target [135]. In particular, highly-curved initial contact tends to result in phagocytosis whereas flatter contact leads to simply spreading with no engulfment. It is then left to the target size to determine whether phagocytosis ever finishes and, if so, how long it takes.

Various groups have examined phagocytosis of spheroids and how uptake compares between spheroids and spheres. For example, Paul et al. measured the total engulfment time for $1.5 \times 1.5 \times 5 \mu \mathrm{m}$ spheroids, finding five times slower uptake than for $3 \mu \mathrm{m}$ spheres (whose surface area is larger) [136]. Also, Sharma et al. examined how oblate and prolate spheroids differ, finding (for targets that are not too large) that oblate spheroids are easier to phagocytose than spheres, which in turn are easier to phagocytose than prolate spheroids [137]. Interestingly, they also found that, despite this, prolate spheroids actually attach to cells better than oblate spheroids, suggesting that their reduced uptake is related not to attachment but to the internalisation process. This shape dependence of attachment has also been addressed by Doshi et al. [138].

Shapes with larger aspect ratios, such as rods and needles, have also been investigated. By using an elegant target fabrication technique called PRINT, Gratton et al. showed that the aspect ratio of rods determines the degree of uptake, with phagocytosis almost completely abolished for sufficiently eccentric particles [139]. This agrees with similar results by Lu et al., who 
found that spheres engulfed easier than rods, which in turn engulfed easier than needles [140]. In addition, Champion and Mitragotri discovered that worm-like particles (long, thin, flexible particles) show almost no phagocytosis [141], agreeing with studies of E. coli filaments [142]. Similarly, Geng et al. determined that short filomicelles (cylindrical micelles) are much more readily phagocytosed than longer filaments [131]. Finally, it is worth noting that these long worm-like shapes may share some similarities with certain bacteria, such as Legionella pneumophila and Borrelia burgdorferi, which are taken up by "coiling phagocytosis", an unusual form of internalisation where a single pseudopod progressively curls around the target $[143,144]$.

Even when target size and shape are fixed, phagocytosis depends on the orientation that the target meets the cell. This tallies with the idea that initiation of phagocytosis is determined by the target curvature at the initial point of contact with the cell [135]. For example, prolate spheroids have been shown to engulf easier when the more highly-curved tip is presented to the cell first [135]. Plausibly this could be related to growth of the nascent actin ring: for tip-first shapes, the actin ring must only grow (and shrink) slowly during engulfment. Similarly, rods are engulfed easier when presented pointed-tip first, with worm-like shapes only phagocytosed when the ends attach to the cell first [141]. In addition, there is evidence that shapes with sufficiently high aspect ratio, such as needles, cannot be engulfed in any orientation [140].

\subsection{Highly-symmetric targets}

Once non-spherical targets are considered, the system typically exhibits lower symmetry, which tends to increase the dimensionality of models. However, this is not the case for sufficiently highly-symmetric targets. In particular, if the target appears circularly symmetric when viewed from above (i.e. its surface can be written as $r=f(z)$ with $z$ the height above the cell) then, at least for non-stochastic models, the entire system (including membrane, receptor density and cytoskeleton) depends only on the radius $r$ and so can be described by a one-dimensional model. Such symmetric targets include spheroids and capped-cylinders, but only when they encounter the cell at precisely the correct orientation. As we discuss below, more general shapes and other orientations require two-dimensional models that also include an angular coordinate.

There are a number of models that examine the effect of particle shape on internalisation, but the majority consider either passive membrane wrapping or endocytosis of relatively small particles $(<500 \mathrm{~nm})$. These typically involve minimising the membrane energy as in $\S 3.3$ and focus on the degree 

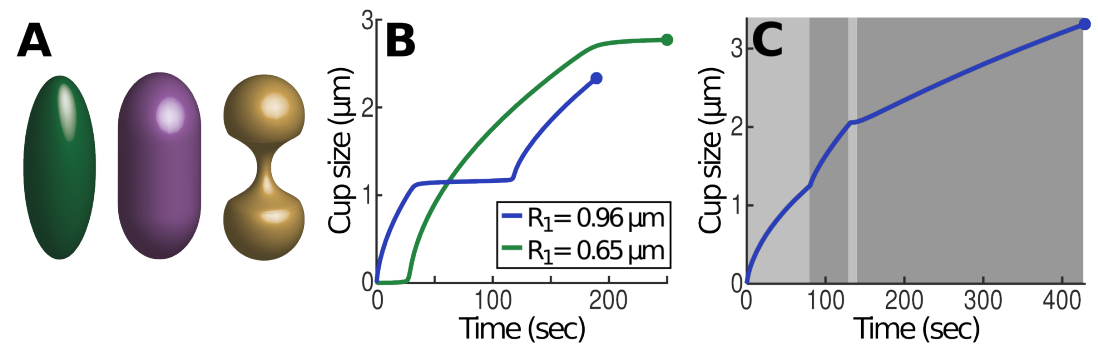

Figure 7. Phagocytosis of non-spherical targets. (A) Example target shapes: spheroid, capped-cylinder and hourglass. (B) Typical cup growth for ellipsoids, with slow-fast-slow engulfment for prolate spheroids (green curve with $R_{1}=0.65 \mu \mathrm{m}$ and $R_{2}=1.13 \mu \mathrm{m}$ ) and fast-slow-fast engulfment for oblate spheroids (blue curve with $R_{1}=0.96 \mu \mathrm{m}$ and $R_{2}=0.49 \mu \mathrm{m}$ ). In each case $R_{2}$ was chosen so that the surface area was equal to that of a sphere with radius $0.8 \mu \mathrm{m}$. (C) Cup growth for a capped-cylinder with $R=0.8 \mu \mathrm{m}$ and $L=0.8 \mu \mathrm{m}$, showing slow-fast-stall-slow engulfment behaviour. Solid circles show the point of full engulfment.

of wrapping $[145,146]$. For example, Dasgupta et al. examined spheroids and rods [147, 148], Váche et al. studied capped-cylinders [149], Tang et al. focused on multiple particles [150], and Decuzzi and Ferrari considered elliptical cylinders [151]. However, these approaches are of only limited relevance to phagocytosis, which involves larger targets and a much more substantial role for the cytoskeleton.

There are far fewer models that directly try to understand shape dependence in phagocytosis. These normally start with one of the above sphericaltarget models, which is then modified to incorporate other target shapes such as spheroids, capped-cylinders and hourglasses (Fig. 7A). In particular, most approaches to date have been based on receptor models, which show that higher-curved regions tend to be harder to engulf, with cup growth no longer square-root in time [80]. Further, sufficiently highly-curved regions often lead to stalling and failed phagocytosis.

Spheroids, which are ellipsoids with two of the semi-major axes the same length, can be described by $\left(x^{2}+y^{2}\right) / R_{1}^{2}+z^{2} / R_{2}^{2}=1$, where $R_{1}$ and $R_{2}$ are the semiprincipal axis lengths. One-dimensional models can only deal with the special case of spheroids aligned so that $\{x, y, z\}=\left\{0,0, \pm R_{2}\right\}$ is the first point to be engulfed. Spheroids are called either prolate (like a rugby ball) if $R_{2}>R_{1}$ or oblate (like a Smartie) if $R_{2}<R_{1}$. Prolate spheroids, where the high curvature is at the top and bottom, exhibit slow-fast-slow engulfment, whereas oblate spheroids, with the high curvature around the middle, 
undergo fast-slow-fast engulfment (Fig. 7B) [80]. Based on this, van Zon et al. argued that incomplete phagocytosis tends to stall at around half engulfment for oblate spheroids and near full engulfment for prolate spheroids [87]. Further, a model that, in addition to receptor diffusion, includes receptor drift and signalling is able to explain why standing ellipsoids engulf quicker than lying-down ellipsoids: since standing ellipsoids reach half engulfment sooner, the second, active stage of engulfment starts earlier, leading to quicker full engulfment [80].

Capped-cylinders, which are sometimes referred to as spherocylinders, are described by a cylinder of length $L$ and radius $R$, capped by hemispheres at both ends (Fig. 7A). They are good models for various rod-shaped bacteria such as E. coli and B. subtilis. When presented tip-first to the cell they show four stages of engulfment with an initial slow stage (due to the highlycurved cap), a fast stage (due to the lower-curved cylinder), a stalled region (whilst the receptor density increases at the cup edge), and a final slow stage (again due to the highly-curved cap) (Fig. 7C) [81]. For constant surface area, capped-cylinders are found to engulf slower than the equivalent spheres, which perhaps explains previous experimental results concerning endocytosis of gold nanoparticles [152].

Other shapes show how complex engulfment behaviour can become. For example, bi-lobed targets (hourglasses) often stall around the narrow neck. This has been seen experimentally in budding yeast [153,154]. Modelling suggests that the precise behaviour in the neck region depends on the exact parameters describing the hourglass, with the quickest engulfment not necessarily corresponding to the smallest neck curvature [81]. Still more complex shapes are expected to show even more subtleties, which is likely to be exploited both by pathogens that try to evade phagocytosis and by those that aim to infiltrate cells.

Finally, recent modelling is allowing the target shape dependence in phagocytosis to be compared to that in other forms of endocytosis. This is based on a receptor-and-signalling model, where the effect of signalling is to recruit actin, which reduces the membrane bending modulus at the cup edge [81]. This could, for example, be due to a change in membrane lipid composition (perhaps caused by recruitment of some GTPase [155]) or increased spontaneous curvature. Non-phagocytic endocytosis is implemented via the same model, but with no actin recruitment and so no change in bending modulus. It is found that engulfment of prolate spheroids (at least in terms of shape rather than size) is similar for phagocytosis and other forms of endocytosis, but that oblate spheroids (which sometimes stall around half engulfment) are easier to completely engulf in phagocytosis. In these oblate cases there is often a pause around half engulfment, whilst sufficient actin 
is recruited to further extend the cup. This approach predicts that phagocytosis not only engulfs larger targets, but is able to engulf a greater range of target shapes than other forms of endocytosis. However, there are always sufficiently eccentric shapes that cannot be engulfed by either process [81].

\subsection{Lower symmetry targets}

One-dimensional models are fundamentally limited by the requirement that targets are rotationally symmetric when viewed from above. Many targets, especially those encountered in nature, do not satisfy this and so require more general approaches. Such cases include both targets that have lower symmetry (such as ellipsoids where all three semi-major axes have different lengths) and high-symmetry targets in non-symmetric orientations (such as cappedcylinders that are lying down relative to the cell surface). Importantly, target orientation dependence can only truly be studied within these more general models. Such models typically consider the full two-dimensional nature of the membrane and allow phagocytic engulfment to proceed at different rates in different directions.

One such approach is based on extending the above receptor models to two dimensions [81]. Conceptually this is trivial, but leads to various biological and numerical complications. The receptor density $\rho(r, \theta, t)$ is now a function not only of the radius $r$ and time $t$, but also the angle $\theta$ around the target (when viewed from directly above the membrane). Similarly, the cup size, $a(\theta, t)$, now also depends on the angular coordinate. Receptor diffusion is described by the full two-dimensional diffusion equation and the rate of cup growth involves taking the normal component of receptor flux at the cup edge. This system is analogous to the two-dimensional supercooled Stefan problem and so can still be thought of as describing the "phase transition" from unbound to bound receptors. With sensible biological assumptions, the boundary conditions are unchanged from the one-dimensional model. However, numerical simulations are now much more involved, with care needed to avoid artefacts that can arise due to instabilities at the cup edge [81].

The cup growth rate now depends on the angle around the target (Fig. 8A). In addition to faster engulfment in lower-curved directions, receptors can also diffuse in the $\theta$-direction. This tends to lead to an "angular amplification" effect whereby directions that engulf quicker than others encourage greater receptor flux towards themselves and so even greater relative engulfment rates. There is no longer a unique definition of half engulfment. For example, the point at which half of the target particle is bound to the membrane (the half-area engulfment time) is generally different to the point when all directions around the cup pass half-way (the half-circumference engulfment 

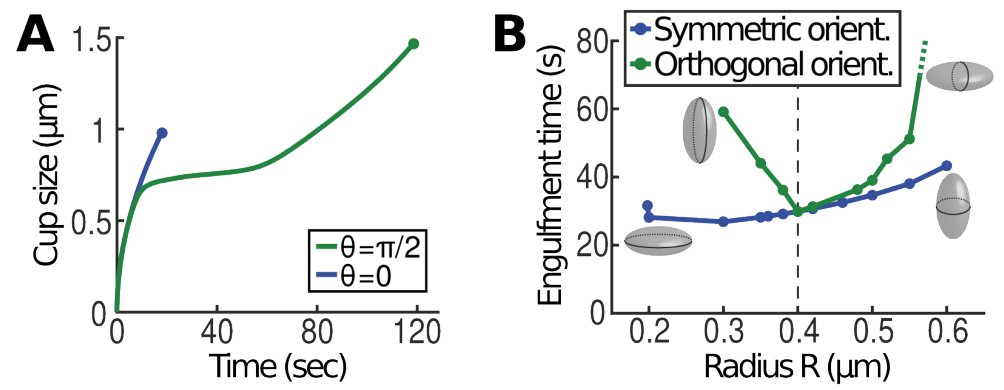

Figure 8. Orientation dependence of spheroidal targets. (A) Cup growth in two orthogonal directions around a lying-down prolate spheroid with $R_{1}=0.3 \mu \mathrm{m}$ and $R_{2}=0.6 \mu \mathrm{m}$. Compared to the direction with constant curvature (blue), the orthogonal direction (green) has both longer arc length and engulfs at a slower rate. (B) The total engulfment time for spheroids of size $\left\{R_{1}, R_{2}\right\}=\{\tilde{R}, R\}$ with $\tilde{R}$ chosen so that the surface area is always equal to that of a sphere of radius $0.4 \mu \mathrm{m}$. The dashed line indicates the spherical case $(R=\tilde{R})$, with oblate spheroids to the left and prolate spheroids to the right. The blue curve represents the rotationally symmetric orientation (when viewed from above) and the green curve the orientation orthogonal to this.

time). Because of this, statements about the degree of engulfment during stalled phagocytosis are more subtle.

Within this framework, it is now possible to examine orientation dependence for spheroids. Perhaps surprisingly, this suggests that prolate and oblate spheroids have completely different engulfment behaviours [81]. As observed experimentally, prolate spheroids engulf quickest when presented tip-first, with sufficiently eccentric targets only engulfing in this orientation. Conversely, the model predicts that oblate spheroids are phagocytosed quickest when the lower curved portion is engulfed first (Fig. 8B). Both these results are explained by the "angular amplification" effect, which leads to the quickest engulfment for the most symmetric orientation. In particular, the shape with the shortest engulfment time (for fixed surface area) is found to be not the sphere but a slightly oblate spheroid presented lying-down, agreeing with the experimental results of Sharma et al. [137].

Another approach to lower symmetry targets uses the above energy-based modelling, which implements a ratchet mechanism driven by stochastic fluctuations, with the membrane shape found by minimising a Helfrich-like energy [51]. This allows a variety of shapes to be investigated, including spheroids and spirals (which are relevant, for example, to various helical 
bacteria such as Helicobacter and Campylobacter). As with receptor-based models, this approach shows that sufficiently highly-curved shapes are not internalised and that tip-first prolate spheroids are engulfed easier than other orientations. Further, spirals are often not engulfed even after twice the time needed for complete engulfment of a sphere of twice the volume [51].

\section{$6 \quad$ Future directions}

Current models of phagocytosis are necessarily simplified in order to obtain mechanistic understanding and testable predictions. As always, this involves a delicate balancing act to ensure that models are of sufficient complexity to capture the real biological processes. For example, current receptor models only consider the role of a single receptor type, whereas multiple different types of receptor will typically be involved for any given target [42]. Further, there is an implicit assumption that there are sufficient receptors within the cup region for a macroscopic density representation (typically governed by the diffusion equation) to make sense. Whether this is always the case is unclear. The complexity of phagocytosis means that intracellular signalling and the role of the cytoskeleton cannot be ignored. However, up until now models have included at most one signalling molecule and simplified approximations of the cytoskeleton (such as actin densities) that ignore the underlying network structure. This is unlikely to be sufficient in the future. Finally, targets have so far been treated as inert particles that never change their orientation during engulfment. Not only is membrane wrapping likely to rotate targets, but living targets (such as bacteria and fungi) often have the ability to control their own shape, position and orientation, meaning that phagocytosis is typically not as simple as the idealised case of ingesting hard plastic beads [7].

Of the numerous outstanding issues in phagocytosis, theoretical-based approaches are likely to be useful in a sizeable proportion. These include questions about force generation (how the phagocytic cup only extends around the edge of targets), the shape of the cup (the factors that control cup thickness and the angle at the cup base), and the role of filopodia in searching for, identifying and capturing targets $[156,157]$. In addition, different targets are initially pushed away from the cell by different distances (the "push-out" distance). Although there is some modelling of this effect for zymosan particles [113], there is little overall understanding of how target and membrane properties interact to control this distance.

Open problems in phagocytosis often separate into either questions about the immune cell or questions about the target particle. Concerning first the cell itself, modelling is best suited to addressing questions related to the cup 
shape and the biophysics of the membrane. For example, there is so far little understanding of the role of membrane makeup (such as lipid composition) during phagocytosis. Theoretical work is ideally suited to tackle such issues via considering spontaneous membrane curvature and cortical tension, both of which have usually been neglected in previous models. Similarly, future modelling should address the sharp angle that is observed between the base of the phagocytic cup and the remainder of the membrane [104], focusing on the extent to which this is influenced by the underlying cytoskeleton. Further, there are numerous unsolved questions regarding the nature of the sharp switch in engulfment rate around half engulfment (see §4) including (i) whether there are always exactly two stages, (ii) whether the switch always occurs at half engulfment, and (iii) the relative speed of each stage. Also, the factors responsible for triggering the switch are still not known, with plausible candidates including the membrane tension [128], the engulfment rate itself and the availability of spare membrane [56].

There are also a multitude of unexplored questions concerning the target particle, exasperated by the tendency for previous work, particularly on the theoretical side, to focus on hard, inert, spherical targets. The time is ripe for considering more realistic, biologically-relevant targets. This includes not only non-spherical shapes and target rotation during engulfment, but also the effect of other target properties such as composition and coating (relevant for certain fungi). In addition, target stiffness is known to influence phagocytic uptake, with softer particles harder to engulf [158]. Models are only now starting to address this topic, although presently focusing on receptormediated endocytosis rather than phagocytosis [159]. Further, ligands need not be distributed uniformly over the target surface and may even move around during engulfment. Dealing with a non-constant density of mobile ligands, not all of which need bind receptors, is likely to be tractable in the coming years with the types of model described here.

Other areas where modelling is likely to aid future progress include the similarities and differences between $\mathrm{Fc} \gamma$ - and CR3-mediated phagocytosis $[43,44]$, frustrated phagocytosis of flat surfaces $[98,128]$, and the role of stochasticity (extending the work of Tollis et al. [51]). Further, the intracellular motion of phagosomes once engulfment is complete, and how this depends on target properties, will require new types of modelling that treat the phagosome as an entity in its own right.

Perhaps one of the most exciting future theoretical aims is to understand the links that phagocytosis shares with other biological processes. These may include not only other types of endocytosis (such as clathrin-mediated endocytosis and macropinocytosis), but also chemotaxis [31], E. coli pedestal formation [160,161], immunological synapse formation [32], and sporulation 
in B. subtilis $[162,163]$. Modelling that focuses on basic biophysical concepts and includes only a few key components is ideal for identifying these fundamental connections between processes that on the surface seem distinct, but that may share ancient evolutionary origins.

Finally, we briefly mention another type of modelling, population based models, that are distinct to the approaches discussed above. Rather than shedding light on phagocytosis at the cellular scale, population models attempt to understand infection at the level of the immune system. There are only a few previous models of this kind, including one by Tran et al. that examined silicosis (a lung disease caused by ingestion of silica particles) [164], and one by Gog et al. that studied the Salmonella infection rate and the possibility that macrophages can be infected by multiple bacteria [165]. These models typically consider a system of equations that describe the number of immune cells $N_{i}$ that contain $i$ ingested particles, with the intention of determining levels of infection and/or the proportion of cells that have no engulfed particles, $N_{0} /\left(N_{0}+N_{1}+N_{2}+\ldots\right)$. Whether such models will play an important role in phagocytosis research is yet to be seen.

\section{Outlook}

The recent progress in understanding the immune system is likely to continue into the foreseeable future. We firmly believe that both traditional purely experimental approaches (without any type of modelling) and purely theoretical methods (that often value mathematical beauty over reality) are misguided. The fact that mathematical modelling of phagocytosis motivated by real data is starting to produce solid, tangible progress demonstrates that a combination of experimental and theoretical techniques will almost certainly be necessary for future progress. Further, models are most useful and predictive when they include only a few key components that are described by simple rules. Although tempting, overly complex models (with too many variables and parameters) are far less successful.

However, despite the progress that theoretical approaches have made, models of phagocytosis (and other parts of the immune system) are still in their infancy. The time is now ripe to investigate more involved models that better capture the intricacies of the process. This probably needs a combination of the different modelling types described in this review, simultaneously drawing on techniques from mathematics, physics and computing, leading to approaches that both unify force, energy and receptor models and simultaneously include the role of actin, signalling and stochasticity. It then seems likely that numerous exciting discoveries await just around the corner. 


\section{Acknowledgments}

DMR and RGE were supported by BBSRC grant BB/I019987/1. DMR was also supported by the Wellcome Trust Institutional Strategic Support Award (WT105618MA) and Medical Research Council Grant MR/P022405/1. RGE also acknowledges funding from ERC Starting Grant 280492-PPHPI.

\section{References}

1. Underhill DM, Ozinsky A (2002) Phagocytosis of microbes: Complexity in action. Annu Rev Immunol 20:825-852.

2. Swanson JA (2008) Shaping cups into phagosomes and macropinosomes. Nat Rev Mol Cell Biol 9(8):639-649.

3. Machta BB, Chachra R, Transtrum MK, Sethna JP (2013) Parameter space compression underlies emergent theories and predictive models. Science 342:604-607.

4. Tauber AI, Chernyak L (1991) Metchnikoff and the origins of immunology: from metaphor to theory. Monographs on the History and Philosophy of Biology, Oxford, UK: Oxford University Press, ISBN 0-19-506447-X.

5. Yutin N, Wolf MY, Wolf YI, Koonin EV (2009) The origins of phagocytosis and eukaryogenesis. Biol Direct 4:9.

6. Stossel TP (1999) The early history of phagocytosis. Advances in Cellular and Molecular Biology of Membranes and Organelles 5:3-18.

7. Celli J, Finlay BB (2002) Bacterial avoidance of phagocytosis. Trends Microbiol 10(5):232-237.

8. Goosney DL, Celli J, Kenny B, Finlay BB (1999) Enteropathogenic Escherichia coli inhibits phagocytosis. Infect Immun 67(2):490-495.

9. Celli J, Olivier M, Finlay BB (2001) Enteropathogenic Escherichia coli mediates antiphagocytosis through the inhibition of PI 3-kinasedependent pathways. EMBO J 20(6):1245-1258.

10. Marchès O, Covarelli V, Dahan S, Cougoule C, Bhatta P, Frankel G, Caron E (2008) EspJ of enteropathogenic and enterohaemorrhagic Escherichia coli inhibits opsono-phagocytosis. Cell Microbiol 10(5):1104-1115. 
11. van Crevel R, Ottenhoff TH, van der Meer JW (2002) Innate immunity to Mycobacterium tuberculosis. Clin Microbiol Rev 15(2):294309.

12. Riber U, Lind P (1999) Interaction between Salmonella typhimurium and phagocytic cells in pigs. Phagocytosis, oxidative burst and killing in polymorphonuclear leukocytes and monocytes. Vet Immunol Immunopathol 67(3):259-270.

13. Massaad MJ, Ramesh N, Geha RS (2013) Wiskott-Aldrich syndrome: a comprehensive review. Ann NY Acad Sci 1285:26-43.

14. Notarangelo LD, Miao CH, Ochs HD (2008) Wiskott-Aldrich syndrome. Curr Opin Hematol 15:30-36.

15. Ochs HD, Thrasher AJ (2006) The Wiskott-Aldrich syndrome. J Allergy Clin Immunol 117(4):725-738.

16. Notarangelo LD, Ochs HD (2003) Wiskott-Aldrich Syndrome: a model for defective actin reorganization, cell trafficking and synapse formation. Curr Opin Immunol 15:585-591.

17. Swanson JA, Johnson MT, Beningo K, Post P, Mooseker M, Araki N (1999) A contractile activity that closes phagosomes in macrophages. J Cell Sci 112:307-316.

18. Diakonova M, Bokoch G, Swanson JA (2002) Dynamics of cytoskeletal proteins during $\mathrm{Fc} \gamma$ receptor-mediated phagocytosis in macrophages. Mol Biol Cell 13(2):402-411.

19. Brown GC, Neher JJ (2012) Eaten alive! Cell death by primary phagocytosis: 'phagoptosis'. Trends Biochem Sci 37(8):325-332.

20. Bonavita E, Galdiero MR, Jaillon S, Mantovani A (2015) Phagocytes as corrupted policemen in cancer-related inflammation. Adv Cancer Res 128:141-171.

21. Dou H, Destache CJ, Morehead JR, Mosley RL, Boska MD, Kingsley J, Gorantla S, Poluektova L, Nelson JA, Chaubal M, Werling J, Kipp J, Rabinow BE, Gendelman HE (2006) Development of a macrophage-based nanoparticle platform for antiretroviral drug delivery. Blood 108(8):2827-2835. 
22. Smith JP (2011) Nanoparticle delivery of anti-tuberculosis chemotherapy as a potential mediator against drug-resistant tuberculosis. Yale J Biol Med 84(4):361-369.

23. Sun T, Zhang YS, Pang B, Hyun DC, Yang M, Xia Y (2014) Engineered nanoparticles for drug delivery in cancer therapy. Angew Chem Int Ed 53:12320-12364.

24. Praetorius NP, Mandal TK (2007) Engineered nanoparticles in cancer therapy. Recent Pat Drug Deliv Formul 1(1):37-51.

25. Kohane DS (2006) Microparticles and nanoparticles for drug delivery. Biotechnol Bioeng 96:203-209.

26. Parveen S, Misra R, Sahoo SK (2012) Nanoparticles: a boon to drug delivery, therapeutics, diagnostics and imaging. Nanomedicine:NBM $8: 147-166$.

27. Pang L, Zhang C, Qin J, Han L, Li R, Hong C, He H, Wang J (2017) A novel strategy to achieve effective drug delivery: exploit cells as carrier combined with nanoparticles. Drug Deliv 24(1):83-91.

28. Lin S-Y, Hsu W-H, Lo J-M, Tsai H-C, Hsiue G-H (2011) Novel geometry type of nanocarriers mitigated the phagocytosis for drug delivery. J Control Release 154:84-92.

29. De Jong WH, Borm PJA (2008) Drug delivery and nano particles: Applications and hazards. Int J Nanomedicine 3(2):133-149.

30. Doherty GJ, McMahon HT (2009) Mechanisms of endocytosis. Annu Rev Biochem 78:857-902.

31. Heinrich V, Lee C-Y (2011) Blurred line between chemotactic chase and phagocytic consumption: an immunophysical single-cell perspective. J Cell Sci 124:3041-3051.

32. Niedergang F, Di Bartolo V, Alcover A (2016) Comparative anatomy of phagocytic and immunological synapses. Front Immunol 7:18.

33. Cannon GJ, Swanson JA (1992) The macrophage capacity for phagocytosis. J Cell Sci 101(Pt 4):907-913.

34. Metchnikoff É (1884) On the relationship of phagocytes to anthrax (Über die Beziehung der Phagocyten zu Milzbrandbacillen). Arch Pathol Anat 97:502-526. 
35. Gordon S (2016) Phagocytosis: The legacy of Metchnikoff. Cell 166(5):1065-1068.

36. Münter S, Kuss C, Meissner M, Frischknecht F (2011) Chapter 2 "Imaging Neutrophil-Pathogen Interactions in vivo". Neutrophils in Infectious Diseases, Bentham Science Publishers, ISBN 978-1-60805382-7.

37. Landecker H (2006) Microcinematography and the history of science and film. Isis 97(1):121-132.

38. Freeman SA, Grinstein S (2014) Phagocytosis: receptors, signal integration, and the cytoskeleton. Immunol Rev 262:193-215.

39. Gordon S (2016) Phagocytosis: An immunobiologic process. Immunity 44(3):463-475.

40. Stuart LM, Ezekowitz RA (2005) Phagocytosis: elegant complexity. Immunity 22(5):539-550.

41. Underhill DM, Goodridge HS (2012) Information processing during phagocytosis. Nat Rev Immunol 12(7):492-502.

42. Flannagan RS, Jaumouillé V, Grinstein S (2012) The cell biology of phagocytosis. Annu Rev Pathol Mech Dis 7:61-98.

43. Kaplan G (1977) Differences in the mode of phagocytosis with Fc and C3 receptors in macrophages. Scand J Immunol 6:797-807.

44. Allen LA, Aderem A (1996) Molecular definition of distinct cytoskeletal structures involved in complement- and Fc receptor-mediated phagocytosis in macrophages. J Exp Med 184:627-637.

45. Le Cabec V, Carréno S, Moisand A, Bordier C, Maridonneau-Parini I (2002) Complement Receptor 3 (CD11b/CD18) mediates type I and type II phagocytosis during nonopsonic and opsonic phagocytosis, respectively. J Immunol 169:2003-2009.

46. Patel PC, Harrison RE (2008) Membrane ruffles capture C3biopsonized particles in activated macrophages. Mol Biol Cell 19:46284639 .

47. Bohdanowicz M, Cosío G, Backer JM, Grinstein S (2010) Class I and class III phosphoinositide 3-kinases are required for actin polymerization that propels phagosomes. J Cell Biol 191:999-1012. 
48. Hall AB, Gakidis MAM, Glogauer M, Wilsbacher JL, Gao S, Swat W, Brugge JS (2006) Requirements for Vav guanine nucleotide exchange factors and Rho GTPases in Fc $\gamma$ R- and complement-mediated phagocytosis. Immunity 24:305-316.

49. Griffin FM, Griffin JA, Leider JE, Silverstein SC (1975) Studies on the mechanism of phagocytosis: I. Requirements for circumferential attachment of particle-bound ligands to specific receptors on the macrophage plasma membrane. J Exp Med 142:1263-1282.

50. Griffin FM, Griffin JA, Leider JE, Silverstein SC (1976) Studies on the mechanism of phagocytosis: II. The interaction of macrophages with antiimmunoglobulin IgG-coated bone marrow-derived lymphocytes. J Exp Med 144:788-809.

51. Tollis S, Dart AE, Tzircotis G, Endres RG (2010) The zipper mechanism in phagocytosis: energetic requirements and variability in phagocytic cup shape. BMC Syst Biol 4:149-165.

52. May RC, Machesky LM (2001) Phagocytosis and the actin cytoskeleton. J Cell Sci 114:1061-1077.

53. Harrison RE, Grinstein S (2002) Phagocytosis and the microtubule cytoskeleton. Biochem Cell Biol 80:509-515.

54. Andrews NL, Lidke KA, Pfeiffer JR, Burns AR, Wilson BS, Oliver JM, Lidke DS (2008) Actin restricts Fc $\epsilon$ RI diffusion and facilitates antigen-induced receptor immobilization. Nat Cell Biol 10:955-963.

55. Mercanti V, Charette SJ, Bennett N, Ryckewaert JJ, Letourneur F, Cosson P (2006) Selective membrane exclusion in phagocytic and macropinocytic cups. J Cell Sci 119:4079-4087.

56. Hallett MB, Dewitt S (2007) Ironing out the wrinkles of neutrophil phagocytosis. Trends Cell Biol 17:209-214.

57. Dewitt S, Hallett M (2007) Leukocyte membrane "expansion": a central mechanism for leukocyte extravasation. J Leukoc Biol 81(5):11601164.

58. Suzaki E, Kobayashi H, Kodama Y, Masujima T, Terakawa S (1997) Video-rate dynamics of exocytotic events associated with phagocytosis in neutrophils. Cell Motil Cytoskel 38:215-228. 
59. Parkin J, Cohen B (2001) An overview of the immune system. Lancet 357:1777-1789.

60. Aderem A (2003) Phagocytosis and the inflammatory response. $J$ Infect Dis 187 Suppl 2:S340-S345.

61. Rabinovitch M (1995) Professional and non-professional phagocytes: an introduction. Trends Cell Biol 5(3):85-87.

62. Malech HL, Deleo FR, Quinn MT (2014) The role of neutrophils in the immune system: an overview. Methods Mol Biol 1124:3-10.

63. Amulic B, Cazalet C, Hayes GL, Metzler KD, Zychlinsky A (2012) Neutrophil function: from mechanisms to disease. Annu Rev Immunol 30:459-489.

64. Rees AJ (2010) Monocyte and macrophage biology: an overview. Semin Nephrol 30(3):216-233.

65. Aderem A, Underhill DM (1999) Mechanisms of phagocytosis in macrophages. Annu Rev Immunol 17:593-623.

66. Reed MC (2004) Why is mathematical biology so hard? Notices Amer Math Soc 51(3):338-342.

67. Mackey MC, Maini PK (2015) What has mathematics done for biology? Bull Math Biol 77(5):735-738.

68. Nowak MA (2006) Evolutionary dynamics: exploring the equations of life. Harvard University Press. ISBN 978-0-67402-338-3.

69. Dorn M, E Silva MB, Buriol LS, Lamb LC (2014) Three-dimensional protein structure prediction: Methods and computational strategies. Comput Biol Chem 53:251-276.

70. Keeling MJ, Rohani P (2007) Modeling Infectious Diseases in Humans and Animals. Princeton University Press. ISBN 978-0-69111-617-4.

71. Tindall MJ, Porter SL, Maini PK, Gaglia G, Armitage JP (2008) Overview of mathematical approaches used to model bacterial chemotaxis I: the single cell. Bull Math Biol 70(6):1525-1569.

72. Tindall MJ, Maini PK, Porter SL, Armitage JP (2008) Overview of mathematical approaches used to model bacterial chemotaxis II: bacterial populations. Bull Math Biol 70(6):1570-1607. 
73. Hodgkin AL, Huxley AF (1952) A quantitative description of membrane current and its applications to conduction and excitation in nerve. J Physiol (Lond) 117:500-544.

74. Swanson JA, Hoppe AD (2004) The coordination of signaling during Fc receptor-mediated phagocytosis. J Leukoc Biol 76:1093-1103.

75. Gao H, Shi W, Freund L (2005) Mechanics of receptor-mediated endocytosis. Proc Natl Acad Sci USA 102(27):9469-9474.

76. Freund LB, Lin Y (2004) The role of binder mobility in spontaneous adhesive contact and implications for cell adhesion. J Mech Phys Solids 52:2455-2472.

77. Stefan J (1891) On the theory of ice formation, particularly in the polar seas (Über die Theorie der Eisbildung, insbesondere über die Eisbildung im Polarmeere). Annalen der Physik und Chemie 42:269286.

78. Meirmanov AM (1992) The Stefan problem. De Gruyter Expositions in Mathematics, Berlin, Germany: Walter de Gruyter, ISBN 3-11011479-8.

79. Gupta SC (2003) The Classical Stefan Problem: Basic concepts, modelling and analysis. North-Holland Series in Applied Mathematics and Mechanics (Book 45), JAI Press, ISBN 978-0-444510860.

80. Richards DM, Endres RG (2014) The mechanism of phagocytosis: two stages of engulfment. Biophys J 107:1542-1553.

81. Richards DM, Endres RG (2016) Target shape dependence in a simple model of receptor-mediated endocytosis and phagocytosis. Proc Natl Acad Sci USA 113:6113-6118.

82. Mooren OL, Galletta BJ, Cooper JA (2012) Roles for actin assembly in endocytosis. Annu Rev Biochem 81:661-686.

83. Smythe E, Ayscough KR (2006) Actin regulation in endocytosis. J Cell Sci 119:4589-4598.

84. Kaur S, Fielding AB, Gassner G, Carter NJ, Royle SJ (2014) An unmet actin requirement explains the mitotic inhibition of clathrinmediated endocytosis. Elife 3:e00829. 
85. Zigmond SH, Hirsch JG (1972) Effects of cytochalasin B on polymorphonuclear leucocyte locomotion, phagocytosis and glycolysis. Exp Cell Res 73:383-393.

86. Axline SG, Reaven EP (1974) Inhibition of phagocytosis and plasma membrane mobility of the cultivated macrophage by cytochalasin B. Role of subplasmalemmal microfilaments. J Cell Biol 62:647-659.

87. van Zon JS, Tzircotis G, Caron E, Howard M (2009) A mechanical bottleneck explains the variation in cup growth during Fc $\gamma \mathrm{R}$ phagocytosis. Mol Syst Biol 5:298-309.

88. Yu C-h, Wu H-J, Kaizuka Y, Vale RD, Groves JT (2010) Altered actin centripetal retrograde flow in physically restricted immunological synapses. PLoS ONE 5:e11878.

89. Garca-Garca E, Rosales C (2005) Adding Complexity to Phagocytic Signaling: Phagocytosis-Associated Cell Responses and Phagocytic Efficiency. Molecular Mechanisms of Phagocytosis, pp58-71, Springer, ISBN 978-0-387-25419-7.

90. Greenberg S (2001) Diversity in phagocytic signalling. J Cell Sci 114:1039-1040.

91. Kwiatkowska K, Sobota A (1999) Signaling pathways in phagocytosis. Bioessays 21(5):422-431.

92. Pratten MK, Lloyd JB (1986) Pinocytosis and phagocytosis: the effect of size of a particulate substrate on its mode of capture by rat peritoneal macrophages cultured in vitro. Biochim Biophys Acta 881(3):307-313.

93. Tabata Y, Ikada Y (1988) Effect of the size and surface charge of polymer microspheres on their phagocytosis by macrophage. Biomaterials $9(4): 356-362$.

94. Koval M, Preiter K, Adles C, Stahl PD, Steinberg TH (1998) Size of IgG-opsonized particles determines macrophage response during internalization. Exp Cell Res 242(1):265-273.

95. Champion JA, Walker A, Mitragotri S (2008) Role of particle size in phagocytosis of polymeric microspheres. Pharm Res 25(8):1815-1821. 
96. Zhang Y, Hoppe AD, Swanson JA (2010) Coordination of Fc receptor signaling regulates cellular commitment to phagocytosis. Proc Natl Acad Sci USA 107(45):19332-19337.

97. Pacheco P, White D, Sulchek T (2013) Effects of microparticle size and Fc density on macrophage phagocytosis. PLoS One 8:e60989.

98. Kovari DT, Wei W, Chang P, Toro J-S, RF Beach, Chambers D, Porter K, Koo D, Curtis JE (2016) Frustrated phagocytic spreading of J774A-1 macrophages ends in myosin II-dependent contraction. Biophys J 111:2698-2710.

99. Hochmuth RM (2000) Micropipette aspiration of living cells. $J$ Biomech 33(1):15-22.

100. Heinrich V, Rawicz W (2005) Automated, high-resolution micropipet aspiration reveals new insight into the physical properties of fluid membranes. Langmuir 21:1962-1971.

101. Herant M, Heinrich V, Dembo M (2005) Mechanics of neutrophil phagocytosis: behavior of the cortical tension. J Cell Sci 118:17891797.

102. Evans E, Yeung A (1989) Apparent viscosity and cortical tension of blood granulocytes determined by micropipet aspiration. Biophys $J$ 56:151-160.

103. Irmscher M, de Jong AM, Kress H, Prins MWJ (2013) A method for time-resolved measurements of the mechanics of phagocytic cups. $J$ $R$ Soc Interface 10(82):20121048.

104. Herant M, Heinrich V, Dembo M (2006) Mechanics of neutrophil phagocytosis: experiments and quantitative models. J Cell Sci 119:1903-1913.

105. Heinrich V (2015) Controlled one-on-one encounters between immune cells and microbes reveal mechanisms of phagocytosis. Biophys $J$ 109:469-476.

106. Dembo M, Harlow F (1986) Cell motion, contractile networks, and the physics of interpenetrating reactive flow. Biophys $J$ 50:109-121.

107. Herant M, Marganski WA, Dembo M (2003) The mechanics of neutrophils: synthetic modeling of three experiments. Biophys $J$ 84(5):3389-3413. 
108. Dembo M (1994) On the free boundary problem of amoeboid motion. Biomechanics of Active Movement and Division of Cells (edited by Akkas N), pp231-283, NATO Advanced Study Institute Series, Berlin, Springer-Verlag, ISBN 978-3-642-78977-9.

109. Pillemer L, Ecker EE (1941) Anticomplementary factor in fresh yeast. J Biol Chem 137:139-142.

110. Di Carlo FJ, Fiore JV (1958) On the composition of zymosan. Science 127:756-757.

111. Lee CY, Herant M, Heinrich V (2011) Target-specific mechanics of phagocytosis: protrusive neutrophil response to zymosan differs from the uptake of antibody-tagged pathogens. J Cell Sci 124:1106-1114.

112. Underhill DM (2003) Macrophage recognition of zymosan particles. $J$ Endotoxin Res 9(3):176-180.

113. Herant M, Lee C-Y, Dembo M, Heinrich V (2011) Protrusive push versus enveloping embrace: computational model of phagocytosis predicts key regulatory role of cytoskeletal membrane anchors. PLoS Comput Biol 7:e1001068.

114. Lee CY, Thompson GR, Hastey CJ, Hodge GC, Lunetta JM, Pappagianis D, Heinrich V (2015) Coccidioides endospores and spherules draw strong chemotactic, adhesive, and phagocytic responses by individual human neutrophils. PLoS One 10(6):e0129522.

115. Helfrich W (1973) Elastic properties of lipid bilayers: theory and possible experiments. Z Naturforsch 28:693-703.

116. Deuling H, Helfrich W (1976) The curvature elasticity of fluid membranes: a catalogue of vesicle shapes. Journal de Physique 37(11):1335-1345.

117. Lipowsky R (1991) The conformation of membranes. Nature 349:475481.

118. Seifert U, Lipowsky R (1995) Morphology of vesicles. Structure and Dynamics of Membranes (edited by Lipowsky $R$ and Sackmann E), pp403-463, Elsevier Science, Amsterdam, North Holland, ISBN 9780-444-81975-8.

119. Seifert U (1997) Configurations of fluid membranes and vesicles. Adv Phys 46(1):13-137. 
120. Yi X, Shi XH, Gao HJ (2011) Cellular uptake of elastic nanoparticles. Phys Rev Lett 107:098101.

121. Ramanan V, Agrawal NJ, Liu J, Engles S, Toy R, Radhakrishnan R (2011) Systems biology and physical biology of clathrin-mediated endocytosis. Integr Biol (Camb) 3(8):803-815.

122. Dmitrieff S, Nédélec F (2015) Membrane mechanics of endocytosis in cells with turgor. PLoS Comput Biol 11(10):e1004538.

123. Seifert U, Langer SA (1993) Viscous modes of fluid bilayer membranes. Europhys Lett 23:71-76.

124. Shkulipa SA, den Otter WK, Briels WJ (2006) Simulations of the dynamics of thermal undulations in lipid bilayers in the tensionless state and under stress. J Chem Phys 125(23):234905.

125. den Otter WK, Shkulipa SA (2007) Intermonolayer friction and surface shear viscosity of lipid bilayer membranes. Biophys J 93(2):423433.

126. Liu J, Kaksonen M, Drubin DG, Oster G (2006) Endocytic vesicle scission by lipid phase boundary forces. Proc Natl Acad Sci USA 103(27):10277-10282.

127. Coelho Netoa J, Agero U, Oliveira DCP, Gazzinelli RT, Mesquita ON (2005) Real-time measurements of membrane surface dynamics on macrophages and the phagocytosis of Leishmania parasites. Exp Cell Res 303(2):207-217.

128. Masters TA, Pontes B, Viasnoff V, Li Y, Gauthier NC (2013) Plasma membrane tension orchestrates membrane trafficking, cytoskeletal remodeling, and biochemical signaling during phagocytosis. Proc Natl Acad Sci USA 110:11875-11880.

129. Champion JA, Katare YK, Mitragotri S (2007) Particle shape: A new design parameter for micro- and nanoscale drug delivery carriers. $J$ Control Release 121:3-9.

130. Nowacek AS, Balkundi S, McMillan J, Roy U, Martinez-Skinner A, Mosley RL, Kanmogne G, Kabanov AV, Bronich T, Gendelman HE (2011) Analyses of nanoformulated antiretroviral drug charge, size, shape and content for uptake, drug release and antiviral activities in human monocyte-derived macrophages. J Control Release 150(2):204-211. 
131. Geng Y, Dalhaimer P, Cai S, Tsai R, Tewari M, Minko T, Discher DE (2007) Shape effects of filaments versus spherical particles in flow and drug delivery. Nat Nanotechnol 2(4):249-255.

132. Mitragotri S, Lahann J (2009) Physical approaches to biomaterial design. Nat Mater 8(1):15-23.

133. Caldorera-Moore M, Guimard N, Shi L, Roy K (2010) Designer nanoparticles: incorporating size, shape and triggered release into nanoscale drug carriers. Expert Opin Drug Deliv 7(4):479-95.

134. Lengerová A, Lenger VJ, Esslová M, Tuscany R, Volfová M (1957) The influence of the shape of dust particles on the rate of phagocytosis in vitro. Br J Ind Med 14(1):43-46.

135. Champion JA, Mitragotri S (2006) Role of target geometry in phagocytosis. Proc Natl Acad Sci USA 103(13):4930-4934.

136. Paul D, Achouri S, Yoon Y-Z, Herre J, Bryant CE, Cicuta P (2013) Phagocytosis dynamics depends on target shape. Biophys $J$ 105(5):1143-1150.

137. Sharma G, Valenta DT, Altman Y, Harvey S, Xie H, Mitragotri S, Smith JW (2010) Polymer particle shape independently influences binding and internalization by macrophages. J Control Release 147(3):408-412.

138. Doshi N, Mitragotri S (2010) Macrophages recognize size and shape of their targets. PLoS One 5(4):e10051.

139. Gratton SE, Ropp PA, Pohlhaus PD, Luft JC, Madden VJ, Napier ME, DeSimone JM (2008) The effect of particle design on cellular internalization pathways. Proc Natl Acad Sci USA 105:11613-11618.

140. Lu Z, Qiao Y, Zheng XT, Chan-Park MB, Li CM (2010) Effect of particle shape on phagocytosis of CdTe quantum dot-cystine composites. MedChemComm 1:84-86.

141. Champion JA, Mitragotri S (2009) Shape induced inhibition of phagocytosis of polymer particles. Pharm Res 26(1):244-249.

142. Möller J, Luehmann T, Hall H, Vogel V (2012) The race to the pole: how high-aspect ratio shape and heterogeneous environments limit phagocytosis of filamentous Escherichia coli bacteria by macrophages. Nano Lett 12(6):2901-2905. 
143. Horwitz MA (1984) Phagocytosis of the Legionnaires' disease bacterium (Legionella pneumophila) occurs by a novel mechanism: engulfment within a pseudopod coil. Cell 36(1):27-33.

144. Rittig MG, Krause A, Häupl T, Schaible UE, Modolell M, Kramer MD, Lütjen-Drecoll E, Simon MM, Burmester GR (1992) Coiling phagocytosis is the preferential phagocytic mechanism for Borrelia burgdorferi. Infect Immun 60:4205-4212.

145. Bahrami AH, Raatz M, Agudo-Canalejo J, Michel R, Curtis EM, Hall CK, Gradzielski M, Lipowsky R, Weikl TR (2014) Wrapping of nanoparticles by membranes. Adv Colloid Interface Sci 208:214-224.

146. Bahrami AH, Lipowsky R, Weikl TR (2016) The role of membrane curvature for the wrapping of nanoparticles. Soft Matter 12(2):581-7.

147. Dasgupta S, Auth T, Gompper G (2013) Wrapping of ellipsoidal nano-particles by fluid membranes. Soft Matter 9:5473-5482.

148. Dasgupta S, Auth T, Gompper G (2014) Shape and orientation matter for the cellular uptake of nonspherical particles. Nano Lett 14(2):687-693.

149. Vácha R, Martinez-Veracoechea FJ, Frenkel D (2011) Receptormediated endocytosis of nanoparticles of various shapes. Nano Lett 11:5391-5395.

150. Tang H, Ye H, Zhang H, Zheng Y (2015) Wrapping of nanoparticles by the cell membrane: the role of interactions between the nanoparticles. Soft Matter 11(44):8674-8683.

151. Decuzzi P, Ferrari M (2008) The receptor-mediated endocytosis of nonspherical particles. Biophys J 94(10):3790-3797.

152. Chithrani BD, Ghazani AA, Chan WC (2006) Determining the size and shape dependence of gold nanoparticle uptake into mammalian cells. Nano Lett 6(4):662-668.

153. Clarke M, Engel U, Giorgione J, Müller-Taubenberger A, Prassler J, Veltman D, Gerisch G (2010) Curvature recognition and force generation in phagocytosis. BMC Biol 8:154.

154. Dieckmann R, von Heyden Y, Kistler C, Gopaldass N, Hausherr S, Crawley SW, Schwarz EC, Diensthuber RP, Côté GP, Tsiavaliaris G, 
Soldati T (2010) A myosin IK-Abp1-PakB circuit acts as a switch to regulate phagocytosis efficiency. Mol Biol Cell 21:1505-18.

155. Hoppe AD, Swanson JA (2004) Cdc42, Rac1, and Rac2 display distinct patterns of activation during phagocytosis. Mol Biol Cell 15(8):3509-3519.

156. Kress H, Stelzer EHK, Holzer D, Buss F, Griffiths G, Rohrbach A (2007) Filopodia act as phagocytic tentacles and pull with discrete steps and a load-dependent velocity. Proc Natl Acad Sci USA 104:11633-11638.

157. Vonna L, Wiedemann A, Aepfelbacher M, Sackmann E (2007) Micromechanics of filopodia mediated capture of pathogens by macrophages. Eur Biophys J 36:145-151.

158. Beningo KA, Wang Y-L (2002) Fc-receptor-mediated phagocytosis is regulated by mechanical properties of the target. J Cell Sci 115:849856.

159. Yi X, Gao H (2017) Kinetics of receptor-mediated endocytosis of elastic nanoparticles. Nanoscale 9(1):454-463.

160. Kaper JB, Nataro JP, Mobley HL (2004) Pathogenic Escherichia coli. Nat Rev Microbiol 2(2):123-140.

161. Goosney DL, de Grado M, Finlay BB (1999) Putting E. coli on a pedestal: a unique system to study signal transduction and the actin cytoskeleton. Trends Cell Biol 9(1):11-14.

162. Ojkic N, López-Garrido J, Pogliano K, Endres RG (2014) Bistable forespore engulfment in Bacillus subtilis by a zipper mechanism in absence of the cell wall. PLoS Comput Biol 10(10):e1003912.

163. Ojkic N, López-Garrido J, Pogliano K, Endres RG (2016) Cell-wall remodeling drives engulfment during Bacillus subtilis sporulation. Elife 5:e18657.

164. Tran CL, Jones AD, Donaldson K (1995) Mathematical model of phagocytosis and inflammation after the inhalation of quartz at different concentrations. Scand J Work Environ Health 21 Suppl 2:5054. 
165. Gog JR, Murcia A, Osterman N, Restif O, McKinley TJ, Sheppard M, Achouri S, Wei B, Mastroeni P, Wood JL, Maskell DJ, Cicuta P, Bryant CE (2012) Dynamics of Salmonella infection of macrophages at the single cell level. $J$ R Soc Interface 9(75):2696-2707. 